NBER WORKING PAPER SERIES

\title{
CONNECTING THE COUNTRYSIDE VIA E-COMMERCE: EVIDENCE FROM CHINA
}

\author{
Victor Couture \\ Benjamin Faber \\ Yizhen $\mathrm{Gu}$ \\ Lizhi Liu \\ Working Paper 24384 \\ http://www.nber.org/papers/w24384 \\ NATIONAL BUREAU OF ECONOMIC RESEARCH \\ 1050 Massachusetts Avenue \\ Cambridge, MA 02138 \\ March 2018
}

\begin{abstract}
We are grateful to the Bill and Melinda Gates Foundation, CEGA, the Clausen Center, the Haas School of Business, the Weiss Family Fund and the Natural Science Foundation of Guangdong Province, China (Grant No. 2020A1515011163) for providing funding for this study. We have benefited from outstanding research assistance by Hero Ashman, Allison Green, Wenwei Peng, Jose Vasquez-Carvajal and Yi Wei. We are grateful to Lijun Sun, Wei Wang, Wei Zheng and Fang Ye for their tireless support during the implementation of this project. We also thank Hongbing Gao, Zhenzhong Sheng, Liang Chen, Wentao Zhang and Zhengwei Jiang for providing us access to and assistance with the transaction database. This research was approved by the Berkeley Office for the Protection of Human Subjects under IRB Protocol No 2015-09-7944 and the research design was pre-registered under RCT ID AEARCTR-0001582. All views expressed and errors are our own. The views expressed herein are those of the authors and do not necessarily reflect the views of the National Bureau of Economic Research.
\end{abstract}

NBER working papers are circulated for discussion and comment purposes. They have not been peerreviewed or been subject to the review by the NBER Board of Directors that accompanies official NBER publications.

(C) 2018 by Victor Couture, Benjamin Faber, Yizhen Gu, and Lizhi Liu. All rights reserved. Short sections of text, not to exceed two paragraphs, may be quoted without explicit permission provided that full credit, including $(\mathrm{C}$ notice, is given to the source. 
Connecting the Countryside via E-Commerce: Evidence from China

Victor Couture, Benjamin Faber, Yizhen Gu, and Lizhi Liu

NBER Working Paper No. 24384

March 2018, Revised April 2020

JEL No. F63,O12,R13

\section{ABSTRACT}

This paper estimates the impact of the first nation-wide e-commerce expansion program on rural households. To do so, we combine a randomized control trial with new survey and administrative microdata. In contrast to existing case studies, we find little evidence for income gains to rural producers and workers. Instead, the gains are driven by a reduction in cost of living for a minority of rural households who tend to be younger, richer and in more remote markets. These effects are mainly due to overcoming logistical barriers to e-commerce, rather than to additional investments to adapt e-commerce to the rural population.

Victor Couture

University of California, Berkeley

Haas School of Business

2220 Piedmont Avenue

Berkeley, CA 94720-1900

couture@berkeley.edu

Benjamin Faber

Department of Economics

University of California, Berkeley

697A Evans Hall

Berkeley, CA 94720

and NBER

benfaber@econ.berkeley.edu
Yizhen $\mathrm{Gu}$

Institute for Economic and Social Research

Jinan University

Guangzhou

China

yizhengu@jnu.edu.cn

Lizhi Liu

Georgetown University

Mcdonough School of Business

37th and O Streets, NW

Washington, DC 20057

United States

lizhi.liu@georgetown.edu 
The number of people buying and selling products online in China has grown from practically zero in the year 2000 to more than 400 million by 2015, surpassing the US as the largest ecommerce market. ${ }^{1}$ Most of this growth has taken place in cities, but the Chinese government recently announced the expansion of e-commerce to the countryside as a national policy priority. The objective is to foster rural economic development and reduce the rural-urban economic divide. $^{2}$ Other developing countries with large rural populations, such as Egypt, India and Vietnam, have recently announced similar e-commerce expansion plans. ${ }^{3}$

These policies have been motivated by a growing number of case studies on highly successful "e-commerce villages" that have experienced rapid output growth by selling both agricultural and non-agricultural products to urban markets via e-commerce. One of the most prominent examples is China: by 2018, the largest e-commerce platform, Taobao, had branded more than 3000 rural market places as "Taobao Villages" based on their high concentration of online sales (AliResearch, 2018). ${ }^{4}$ Inspired by these success stories, much of the current policy focus has been on rural producers. By lowering trade and information costs to urban markets, e-commerce is meant to raise rural incomes through higher demand for local production, better access to inputs and stronger incentives for rural entrepreneurship. There has been less emphasis on the potential benefits to rural consumers. However, recent descriptive evidence from urban China suggests that e-commerce demand is strongest in smaller and more remote cities, pointing to potentially large consumer gains in rural areas. ${ }^{5}$

The recent growth of e-commerce in a number of rural markets has captured the imagination of policy-makers, but important questions remain about whether market integration through online trading platforms can have a broad and significant impact on rural development. There is also little available evidence on the characteristics of households and markets that may benefit more or less from e-commerce, and on the effectiveness of investments targeted at lifting different types of barriers to rural e-commerce access. ${ }^{6}$ To answer these questions, this paper studies the first nationwide e-commerce expansion program. From 2014 to 2018, this program connected more than 40,000 Chinese villages to e-commerce. Our analysis combines a randomized control trial (RCT), that we implement across villages in collaboration with a large Chinese e-commerce firm, with a new collection of household and store price survey microdata and the universe of transaction records from the firm's internal database.

E-commerce is the ability to buy and sell products through online transactions coupled with

\footnotetext{
${ }^{1}$ In both number of users and total sales. See e.g. PFSweb (2016) and Statista (2016).

${ }^{2}$ The policy agenda of "Alleviating Poverty through E-Commerce" has featured in the government's "No.1 Central Document" each year since 2014.

${ }^{3}$ See e.g. Egypt's "National E-Commerce Strategy" (MCIT, 2016), “Digital India" (MEITY, 2016), Vietnam's “E-Commerce Development Masterplan" (PM, 2016) and UNCTAD's new technical assistance platform "eTrade For All: Unlocking the Potential of E-Commerce in Developing Countries" (UNCTAD, 2016).

${ }^{4}$ See e.g. World Bank publications by Luo \& Niu (2019) and Luo et al. (2019). E-commerce villages have also received widespread media attention (e.g. "China's Number One E-Commerce Village" (BBC Global Business, 01 May 2013), “Inside China's Tech Villages" (The Telegraph, 05 Nov 2016), “Once Poverty-Stricken, China's Taobao Villages Have Found a Lifeline" (QZ, 01 Feb 2017), “Taobao Villages Are Turning Poor Communities into Huge Online Retail Hubs" (Business Insider, 27 Feb 2017)).

${ }^{5}$ In the US, the share of e-commerce in 2015 retail sales was about 10-15 percent (e.g. FRED (2016)). In China, McKinsey (2016) reports this share to be as high as 20-30 percent in smaller cities, and Fan et al. (2016) find this share increase by 1.2 percentage points as city population decreases by 10 percent.

${ }^{6}$ These questions complement the recent literature on the consumer gains from e-commerce in the US (e.g. Brynjolfsson et al. (2003); Goldmanis et al. (2010); Dolfen et al. (2017)).
} 
transport logistics for local parcel delivery and pickup from producers. Bringing e-commerce to the countryside in developing countries requires more than internet access. The internet is already available in most of the Chinese countryside due to both smartphones and expanding broadband access. Instead, there are two current barriers to rural e-commerce trading, which we refer to as the logistical and the transactional barriers. The logistical barrier relates to the lack of modern commercial parcel delivery services. These providers already operate distribution networks across Chinese cities, but have not entered large parts of the countryside. One well-known challenge to rural transport logistics is the so-called "last mile" between urban logistical hubs and small pockets of rural population. The transactional barrier refers to the potential lack of familiarity with navigating online platforms or access to online payment methods that rural households may face. Villagers may also not trust transactions that occur before inspecting the product or without interacting with buyers in person.

To overcome these barriers, the Chinese government recently partnered with a large firm that operates a popular Chinese e-commerce platform. The program aims to invest in the necessary transport logistics to offer e-commerce in rural villages at the same price, convenience and service quality that buyers and producers face in their county's main city center. To this end, the e-commerce firm builds warehouses as logistical nodes for rural parcel delivery/pickup near the urban center, and fully subsidizes transport between the county's city center to and from the participating villages. To address additional transactional barriers specific to the rural population, the program installs an e-commerce terminal in a central village location. A terminal manager employed by the firm is available to assist villagers in buying and selling products through the firm's e-commerce platform. Villagers can pay upon receipt of their products, or get paid upon pickup of their shipments in cash at the terminal location. The terminal is available in addition to the platform's online app-based interface for buying and selling.

An advantage of this setting is that we can study the reduction in trading frictions through e-commerce without confounding the counterfactual with the effects of first-time internet access or reductions in transport costs more broadly. The participating villages were already connected to the internet, and the program makes no changes on this front. Furthermore, the program only directly affects trading partners through e-commerce, while other trade costs, e.g. to control villages, remain unchanged. ${ }^{7}$ The RCT and data analysis that we describe below exploit this empirical setting to provide evidence on the local economic effects of e-commerce trading access on rural households. ${ }^{8}$ In addition to evaluating the program's overall impact, we use the features of this setting to provide evidence on the relative importance of trade cost reductions (logistical barrier) and additional investments targeted at adapting e-commerce to the rural population (transactional barrier).

The analysis proceeds in two steps. In the first step, we randomize the arrival of e-commerce across 100 villages in 3 provinces and 8 counties, and use our survey microdata to estimate the

\footnotetext{
${ }^{7}$ In this way we relate but also differ from existing literature on the effects of transport cost reductions on rural markets (e.g. Van de Walle (2009); Casaburi et al. (2013); Asher \& Novosad (2018)) and on the effects of the internet on rural markets (e.g. Chapman \& Slaymaker (2002); Goyal (2010); Forman et al. (2012); WB (2016)). The empirical context and RCT allow us to study a different counterfactual of recent policy interest.

${ }^{8}$ We do not also attempt a social cost-benefit analysis of this program, which would require additional detailed and confidential information on the cost side from both the e-commerce firm and local and national governments, to which we do not have access.
} 
impact on local economic outcomes. We then bring to bear the firm's internal database covering the universe of transactions for about 12,000 villages in 5 provinces where the program had entered by April 2017. These data allow us to provide additional evidence on a number of questions outside the scope of the fieldwork. In particular, we investigate whether consumption or production-side effects take longer to materialize than the 12-month window we are able to study in the experiment, and whether our household survey data may have missed rare but highly successful tail events on the producer side.

We interpret these results through the lens of a simple theoretical framework to quantify their implications for household welfare. We find no evidence of significant gains or losses on the production and income sides of the local economy. This finding remains when using the firm's database to quantify village out-shipments up to 2.5 years after program arrival and using the universe of transaction records instead of survey samples. Instead, we find that the gains from ecommerce are driven by a reduction in cost of living for retail consumption. This effect is sizable (5 percent) among the group of rural households who are induced to use the new e-commerce option. These users, however, only represent about 15 percent of rural households, who are on average richer, younger and living in more remote markets. In terms of channels, we find that the gains are concentrated among villages that were not previously serviced by commercial parcel delivery, suggesting the program's effects are mainly due to overcoming the logistical barrier, rather than additional investments to lift transactional barriers specific to rural households. Consumer gains are strongest for durable product groups, such as electronics and appliances. We also find suggestive evidence of additional product variety in local stores, from sourcing new products through e-commerce. However, we find no evidence of pro-competitive effects on local store prices for pre-existing merchandise.

Overall, our findings put into context the transformative effects of e-commerce on rural markets that have been documented in numerous case studies on "e-commerce villages" in China and elsewhere. Our results suggest that such success stories are not representative of the countryside as a whole, and should not be used as a guide to set policy expectations. Adding to this insight the significant heterogeneity that we document on the consumption side, access to e-commerce appears to offer economic gains to certain groups of the rural population and in certain places, rather than being broad-based. As this evidence is based on one of the first and so far largest ecommerce expansion policies in the developing world, these findings are particularly relevant for the growing number of governments who have recently announced similar plans using China as a blueprint. ${ }^{9}$ In this light, we hope that our work inspires future research aimed at investigating the local factors and potential complementary interventions, such as e.g. business training for e-commerce or access to credit, that enable certain groups and places to reap the gains from trade through e-commerce.

\section{Experimental Design and Data}

The experiment takes place in 8 counties located in Anhui, Henan and Guizhou provinces. The unit of randomization is the village. For each county, we obtain a list of villages where the

\footnotetext{
${ }^{9}$ In addition to the country plans discussed above, e.g. Thailand's recent "Smart Village" program has been designed based on field visits to Taobao Villages in China ("Commerce Ministry Touts Taobao Village Model" (Bangkok Post, 24 Dec 2018)).
} 
firm plans to introduce the e-commerce program. We ask the firm to extend this list by 5 suitable village candidates in the county that would not have been part of the list in the absence of our research. We then randomly select 5 control villages and 7-8 treatment villages per county from this extended list. The remaining villages receive the e-commerce program as planned. The full sample in which we collect survey data thus includes 40 control villages and 60 treatment villages, randomly selected from 432 village candidates. Compliance with our assignments is not complete: the program was rolled out in 38 of the 60 treatment villages and in 5 out of the 40 control villages. We therefore report both intent-to-treat and treatment-on-treated effects. The main reason for imperfect compliance is that we are able to randomize treatments before the terminal manager applicants receive job offers, and some candidates end up rejecting. ${ }^{10}$ Finally, in one of the counties, the local government suspended our team's data collection mid-way, leaving 4 of the 100 villages without endline data. The online appendix provides additional details, maps and descriptive statistics discussed below.

\section{Household Survey Data}

For the baseline survey at the end of 2015 and beginning of 2016, we collect data from 28 households per village. 14 of those households are randomly sampled within a 300 meter radius of the planned terminal location ("inner zone"), and 14 households are randomly sampled from other parts of the village ("outer zone"). The second round of data collection occurs one year after the baseline. ${ }^{11}$ We collect data from the same households as in the first round, and were also able to extend the original sample by 10 randomly sampled households within the inner zone. We collect detailed information about household retail consumption expenditures split across 9 categories and for production inputs. We also collect information on household incomes, hours worked, occupations and sectors of different members, asset ownership, financial accounts, internet use, and migration.

The median age of all household members in the baseline survey is 44 and the median household size is 3 . The primary earner is a farmer in 60 percent of households, and 82 percent of them completed at least primary school. Rural households are significantly poorer than in urban China: mean monthly income and retail expenditure per capita are about RMB876 and RMB732 respectively. 80 percent of primary earners work inside the village. However, on average half of household retail expenditures occur outside the village, requiring a round-trip to the nearest township center that takes on average one hour. Close to 40 percent of households report having used the internet, more than 50 percent own smartphones and close to 30 percent report owning a laptop or PC. Almost all households own a TV. At the same time, e-commerce penetration is very limited compared to urban regions: both the average share of household retail expenditure on e-commerce deliveries and the share of revenues from online selling in monthly income are less than 1 percent. Neither of these change over time in the endline survey among control villages.

\footnotetext{
${ }^{10}$ Incomplete acceptance rates are standard in this setting and unrelated to the experiment (as applicants were unaware).

${ }^{11}$ The fast pace of the program's expansion places bounds on the timing of the endline. Our control villages ranked highly when the firm decided to launch additional waves of program expansion that were rolled out shortly after the endline.
} 


\section{Local Retail Price Survey Data}

We aim to collect 115 price quotes in each village. We sample products across 9 retail consumption categories based on expenditure shares of rural households in Anhui and Henan from the 2012 China Family Panel Study (CFPS). We also include production and business inputs. We sample stores to be representative of local retail outlets (stores and market stalls). In villages with few stores, we sample all of them. We sample products within stores to capture a representative selection of locally purchased items within that store and product group. Each price quote is at the barcode-equivalent level where possible (recording brand, product name, packaging type, size, flavor if applicable). In the endline survey, we collect price quotes of the same products and retail outlets. In cases of either store closure or product disappearance, we include a new price quote within the same product category. The median number of sampled stores is 3 per village. The median floor space is $50 \mathrm{~m}^{2}$, and the median store has not added new products within the last month.

\section{Firm's Admin Database}

We complement the survey data with administrative records from two different divisions of the firm covering 5 provinces (the three RCT provinces plus Guangxi and Yunnan where the firm was also active). The first database covers the universe of e-commerce purchases made through the program in every participating village from November 2015 to April 2017. The data cover approximately 27.3 million transaction records across 12,000 villages over this period. For each transaction, the database contains information about the product category, number of units, amount paid and a unique buyer identifier. Given that many villages had already been in operation for several months prior to November 2015, these data cover adjustment periods beyond the 12-months window that our RCT captures: transactions are observed up to two years and 4 months post-installation. The second database covers the universe of sales transactions, i.e. outshipments from the villages, through the firm's distribution network for the same universe of roughly 12,000 villages in the 5 provinces from January 2016 to April 2017. For each transaction, the database records the village of origin and the weight of the out-shipment in kilograms $(\mathrm{kg})$. The total number of e-commerce out-shipments over this period is roughly 500,000.

\section{Analysis}

\subsection{Evidence from Survey Data}

We run regressions of the following form:

$$
y_{h v}^{\text {Post }}=\alpha+\beta_{1} \text { Treat }_{v}+\gamma y_{h v}^{\text {Pre }}+\epsilon_{h v},
$$

where $y_{h v}$ is an outcome of interest for household $h$ living in village $v .^{12}$ For outcomes from the retail price data, $h$ indexes individual price quotes or store-level outcomes instead. Treat $t_{v}$ is either an indicator of randomly assigned treatment status when estimating the intent-to-treat effect (ITT) or actual treatment status when estimating the treatment-on-the-treated effect (TOT) and instrumenting with intended treatment. We cluster standard errors at the level of the treatment (village level), and report point estimates both individually and after combining outcomes into

\footnotetext{
${ }^{12}$ While improving precision, none of the significant findings below rely on the inclusion of baseline outcomes $y_{h v}^{P r e}$.
} 
category indices following Kling et al. (2007) (“K-L-K”).

Table 1 presents estimation results for the average effects on household consumption (Panel A), incomes (Panel B) and local retail prices (Panel C). Our discussion here focuses on the TOT results. On the consumption side, we find that the program leads to an uptake of on average 9 percent of households using the new e-commerce option in treatment villages compared to control villages. As documented by the non-zero mean among control villages, this effect masks additional uptake due to users in nearby control villages, increasing the effect on uptake to about 14 percent of village households. We further investigate such spillovers at the end of this section. The treatment effect on the new option's share in total household retail expenditure is 1.24 percent for the average village household. Thus, households that report having used the e-commerce option spent on average $0.0124 / 0.089=14.1$ percent of their retail consumption during the past month. We find stronger effects on durables compared to non-durables: for durables, the share of household expenditure is 6.9 percent for the average household, indicating a 45 percent shift in durable consumption to the new e-commerce option among uptaking households. ${ }^{13}$ For non-durables, the treatment effect on the share of household retail expenditure is 1 percent for the average household, indicating that ever-users spend on average about 11 percent of total non-durables expenditure on the new e-commerce option. While households do shift part of their expenditures to e-commerce, there are no significant treatment effects on total monthly retail expenditures. The last column of Panel A combines 11 outcomes related to substitution into e-commerce into a single index, defined as the equally weighted average of $z$-scores that are calculated by subtracting the mean and dividing by the standard deviation of the control group. The treatment effect on this index is 0.89 and significant at the one percent level. ${ }^{14}$

Panel B reports point estimates on incomes per capita that are close to zero and not statistically significant. As above, we also report a single income-related index combining 14 outcomes related to income generation. We find no effects on either annual or monthly incomes, from agricultural or non-agricultural sources, on labor supply as measured by hours worked by the primary (or secondary) earner or on online selling activity, online revenues, sourcing of business inputs or business creation offline or online. In terms of precision, the ITT point estimate on the income index indicates detectable positive effects down to about 2.6 percent of a standard deviation (one-sided 95\% CI).

In Panel C, we find no significant reduction in local store prices for continuing products that we observe in the same local retailer in both baseline and endline data. The point estimate is close to zero and positive, and not statistically significant. Given our sampling framework, the unweighted average effect on local retail prices is akin a Laspeyres price index for local retail consumption. We also find no effect when combining 4 outcomes related to local retail prices and product exit/additions into a single index. We find one piece of evidence suggestive of knock-on effects on pre-existing local stores. The effect on the number of new products per store over the past month is 4 goods and is significant at the $10 \%$ level.

\footnotetext{
${ }^{13}$ For households who purchase durables over the past three months, the treatment effect on uptake is 15.3 percent instead of 9 percent. This yields an effect on the average durables consumption share among uptakers of $0.069 / 0.153=45$ percent.

${ }^{14}$ See Appendix B for details on the K-L-K indices in Table 1.
} 


\section{Heterogeneity}

In Table 2 we explore the heterogeneity of these effects. We begin by investigating the effect of the program as a function of pre-existing availability of commercial parcel delivery at the village level. Villages serviced by commercial parcel delivery operators during our baseline survey already had access to local e-commerce deliveries. Interacting the treatment with pre-existing parcel delivery status therefore allows us to shed light on the combined effect of removing both logistical and transactional barriers (among villages without pre-existing parcel delivery), from the effect of removing only the transactional barrier (adding a terminal interface in villages with pre-existing parcel delivery). ${ }^{15}$ Next, we investigate heterogeneity across a basic set of household demographics that have been documented in recent studies of internet and e-commerce use in China (respondent age, education and income per capita) (CNNIC, 2015a,b). We also consider residential distance to the planned terminal location and a measure of village remoteness (motivated by Fan et al. (2016)) based on road travel distance to the nearest township center. One should note that these interaction terms are not causally identified by experimental variation and provide additional suggestive evidence.

We first run regressions in which one characteristic at a time is interacted with the treatment, then a combined regression with all interactions included jointly. On the consumption side, we find that the effect on program uptake is driven by villages that were not initially connected to commercial parcel delivery services. The treatment effect is 10.6 percent among the roughly 85 percent of villages not previously connected to commercial parcel delivery, but a relatively precise zero for villages with pre-existing parcel delivery. On the production and local retail sides, we find no significant effects in either group of villages, confirming the earlier pooled results. ${ }^{16}$ Turning to other potential sources of heterogeneity, we find that younger, richer households who live in closer proximity to the planned terminal, and in more remote villages experience stronger uptake on the consumption side. For example, consumption uptake would close to double if average incomes were to double and primary earners were on average 10 years younger. Somewhat surprisingly, we find no significant heterogeneity with respect to the years of education.

\section{Spillovers}

We investigate the role of spillovers that could bias findings from the survey data. For example, if trade linkages with surrounding villages are an important driver of the local economy, then the comparison between treated and control villages could miss income or retail price effects. More simply, residents in control villages could use e-commerce terminals in a nearby treated village. To investigate these forces, we follow Miguel \& Kremer (2004) and use variation in a village's exposure to other nearby treated villages after controlling for proximity to all villages. ${ }^{17}$ On the consumption side, we find evidence of positive spillovers from nearby terminals in other villages, as previewed above. In contrast, we find no evidence of cross-village spillovers on retail stores or on the production side. Consistent with the absence of income or price spillovers, we

\footnotetext{
${ }^{15}$ The transport subsidy does not affect villages previously serviced by parcel delivery, as logistics operators offered service in a few rural locations at the same rate as elsewhere in the county prior to program entry.

${ }^{16}$ In line with the pooled results, Appendix A reports some evidence that effects on product additions and stores sourcing online are stronger in villages without pre-existing parcel delivery.

${ }^{17}$ See Online Appendix C.
} 
also confirm in microdata from the 2010 Census that the fraction of village market access driven by trade with other nearby rural markets is minor (less than 3 percent). ${ }^{18}$

\subsection{Evidence from Firm Database}

We use the firm's internal transaction database to provide evidence on two questions that are outside the scope of the fieldwork. ${ }^{19}$ First, to what extent are consumption and production responses to e-commerce access increasing beyond our survey's 12-month time window? Second, are our survey data missing rare but highly successful tail events on the production side that could shift the average effect on local household incomes?

To answer these questions, we use the universe of transaction records from 5 provinces and about 12,000 villages that had been treated by April 2017 to estimate the following event study specification:

$$
y_{v m}=\theta_{v}+\delta_{m}+\sum_{j=-3}^{24} \beta_{j} \text { MonthsSinceEntry } y_{j m}+\epsilon_{v m} .
$$

where $v$ indexes villages, $\delta_{m}$ is a set of month fixed effects between November 2015 and April $2017, \theta_{v}$ is a village fixed effect. Each observation in equation (2) is a village in a given month. $y_{v m}$ is one of 4 village-level monthly outcomes: number of buyers, number of purchase transactions, number of out-shipments and total weight of out-shipments in $\mathrm{kg}$. We create a balanced panel in the sense that each of the villages appears once per month in the panel, for each of the 18 months for which we have data (16 months in the shipment data). This spans terminal observations of up to 17 months pre-installation for villages connected in April 2017, and up to 28 months postinstallation for the earliest villages connected by the program. A negative index $j$ denotes the number of months prior to program entry. A positive $j$ indexes the number of months since the program started operation, so $\beta_{0}$ is a measure of average outcomes for villages during the month of their installation, $\beta_{1}$ captures averages one month after installation, and so on. We assign an index of $j=24$ to all observations equal or beyond 24 months after program entry, so $\beta_{24}$ captures average outcomes among villages that have been in operation for more than two years. Each of the $\beta_{0}-\beta_{24}$ are estimated relative to the omitted category that are periods pre-program entry (zeros by construction since the program did not exist).

Figure 1 presents the event-study plots for village-level outcomes on the consumption and production sides. On the consumption side, we find little evidence of increasing uptake past our survey's one-year timeline. Program usage increases rapidly for about 2 to 4 months after opening, and then plateaus at around 85 buyers and 280 transactions per month per village. On the production side, we find evidence that the number and total weight of out-shipments increase smoothly over time after program entry, and beyond the 12-month window that we cover in our survey data. The effect increases by roughly 50 percent when comparing the point estimate on the total weight of out-shipments 12 months post-entry to that more than 2 years post-entry. These results suggest that production-side adjustments take longer to fully materialize than our

\footnotetext{
${ }^{18}$ Given how small villages are compared to cities, and that a small fraction of all villages participate in the program, GE effects on urban centers are unlikely in our setting.

${ }^{19}$ Online Appendix D also uses these data to investigate the representativeness of our RCT village sample and the timing/seasonality of the survey data collection.
} 
survey's 1-year horizon. Despite this positive trend, the average monthly estimated effects at the village level remain small more than two years post implementation, at around 10 out-shipments with a combined weight of $30 \mathrm{~kg}$.

Turning to the second question, our sampling of 38 households per village in the survey data collection may be insufficient to capture rare but very successful events on the production side. To investigate this issue, we use the universe of out-shipments depicted in Figure 1 and make the following assumptions to get an upper-bound estimate for these shipments' potential income creation in the local village economy: we assume i) that the entire value of these shipments is local value-added, and ii) that the average value per $\mathrm{kg}$ of these shipments is as high as that of Chinese exports to the rest of the world. ${ }^{20}$ Even under these assumptions, we find that e-commerce out-shipments account for on average at most a 0.17 percent increase in local income per capita more than 2 years after the program's arrival. To conclude, this average longer-term effect-that we can estimate precisely in Figure 1 using the firm's transaction data-would still be consistent with the statistical zero results on incomes and the production side that we find using the RCT survey data after one year. ${ }^{21}$

\section{Evaluation}

In the final part, we interpret the program's observed effects through the lens of a simple theoretical framework. The most robust effect that we find is on the substitution of local households' retail expenditures to the new e-commerce shopping option. To quantify the cost of living implications consistent with these estimates, we follow a revealed-preference approach as in recent work by Atkin et al. (2018) and structure household preferences into three tiers: the upper tier is Cobb-Douglas over broad product groups $g \in G$ (durables and non-durables) in total consumption, the middle tier is CES across retailers $s \in S$ selling that product group (e.g. local stores, market stalls or the e-commerce option), and the final tier is across individual products within groups $b \in B_{g}$ which can be left unspecified. ${ }^{22}$ The direct consumer gains from the arrival of the e-commerce option, measured as a percentage of initial household expenditure, can then be expressed as follows:

$$
\frac{\text { Gains }_{h}}{\text { Initial Expenditure }_{h}}=\prod_{g \in G}\left(\left(\sum_{s \in S_{g}^{C}} \phi_{g s h}^{1}\right)^{\frac{1}{\sigma_{g}-1}}\right)^{\alpha_{g h}}-1,
$$

where $\sigma_{g}$ is the elasticity of substitution across retail options to source consumption in product group $g, \alpha_{g h}$ is the initial expenditure share on that product group for household group $h$ and $\sum_{s \in S_{g}^{C}} \phi_{g s h}^{1}$ is the share of retail expenditures that is not spent on the new e-commerce option postintervention (where $s \in S_{g}^{C}$ indexes continuing local retailers and $\phi_{g s h}^{1}$ is the endline expenditure share on retailer $s$ in product group $g$ of household group $h$ ).

To estimate this expression, we require information about the program's effect on $\sum_{s \in S_{g}^{c}} \phi_{g s h}^{1}$

\footnotetext{
${ }^{20}$ On average RMB66.5 per $\mathrm{kg}$ in 2015 and 2016 (WITS database).

${ }^{21}$ Related to this, much of the existing literature on ICT in developing countries have estimated effects after relatively short periods: e.g. Jensen (2007) documents significant effects of Indian cell phone towers on market prices and other outcomes within weeks post-installation. More recently, Hjort \& Poulsen (2018) document effects of fast-speed internet on local employment and incomes in Africa that arise within 3-12 months post-installation.

${ }^{22}$ See Online Appendix E for more details.
} 
and the parameters $\alpha_{g h}$ and $\sigma_{g}$. For the $\alpha_{g h}$, we use our baseline data on household expenditure shares across product groups. For ex-post expenditure shares on the new e-commerce option, we use the treatment effects among the 85 percent of villages without pre-existing parcel delivery connections reported in Table 2. These villages experienced the removal of both logistical and transactional barriers to e-commerce trading. We include mean program usage among control villages in these treatment effects to account for program spillovers as discussed above.

We perform this welfare computation for two different groups of local households. First for the average sample household, for whom the average effect on the terminal share of total retail consumption is 1.6 percent, and second for households who report ever having used the terminal for consumption, for whom this effect is 14 percent. We also estimate price index effects separately for durable and non-durable consumption. And we report estimates both with and without re-weighting households according to sampling weights. Finally, we calibrate $\sigma_{g}$ using estimates from Atkin et al. (2018) for households in Mexico with incomes comparable to those of rural Chinese households in our survey ( $\sigma_{N}=3.87$ for non-durables and $\sigma_{D}=3.85$ for durables).

Table 3 reports the estimation results. The average reduction in retail cost of living among households who experienced the lifting of both logistical and transactional barriers is 0.82 percent. This effect increases to 5.6 percent among the roughly 15 percent of households who ever used the new e-commerce option. These effects are slightly lower at 0.73 and 4.7 percent respectively when weighting our sample households to represent the average population living in these villages. Underlying these effects are strong consumer gains in durable consumption: 3 percent for the average village household and 16.6 percent among users. For reference, retail consumption across all product groups accounts for on average 55 percent of total household expenditure among the rural households in the sample. ${ }^{23}$

Finally, to investigate the distribution of these gains, we use treatment effects from the joint heterogeneity specification in the bottom panel of Table 2. We estimate this specification with the dependent variable being the household expenditure share on the new e-commerce option for either durables or non-durables. For each sample household in treatment villages without pre-existing parcel delivery, we then compute a fitted value of the effect on $\sum_{s \in S_{g}^{C}} \phi_{g s h}^{t 1}$, based on the primary earner's age, income per capita, residential distance to the planned terminal and distance to the nearest township center (remoteness), included jointly. Figure 2 shows these graphs. Ranking households along each of these dimensions, we find more than 4-fold differences in the price index effect within the sample. For example, the average rural household with a 25 yearold primary earner experiences a reduction in retail cost of living of about 1.5 percent (without conditioning on uptake), which drops below 1 percent past the age of 40 and close to zero past the age of 60 .

Overall, our findings suggest that the welfare gains from e-commerce trading access are limited to certain groups of rural households and particular markets, rather than being broad-based. First, we show that the income and production-side effects that have been the focus of the existing literature on "e-commerce villages" are not representative of the countryside, even when focusing on a sample of rural markets in the RCT that were chosen by the firm for successful e-commerce

\footnotetext{
${ }^{23}$ We also evaluate robustness to alternative $\sigma_{g}$. Assuming $\sigma_{N}=2.87$ and $\sigma_{D}=2.85$ yields larger gains (a 1.27 percent reduction in retail cost of living on average and 8.74 percent among users). Assuming $\sigma_{N}=4.87$ and $\sigma_{D}=4.85$ yields slightly smaller effects ( 0.61 and 4.12 percent respectively).
} 
expansion. Second, we find strong heterogeneity in the consumer gains from e-commerce across villages and households within them. In this light, we hope this work can inspire additional research to investigate what types of local factors or complementary interventions allow rural markets to reap the gains from trade through e-commerce for both producers and consumers.

\section{References}

AliResearch. (2018). China's taobao villages. Alibaba Research Report.

Asher, S., \& Novosad, P. (2018). Rural roads and local economic development. Working Paper, Dartmouth College.

Atkin, D., Faber, B., \& Gonzalez-Navarro, M. (2018). Retail globalization and household welfare: Evidence from mexico. Journal of Political Economy.

Brynjolfsson, E., Hu, Y., \& Smith, M. D. (2003). Consumer surplus in the digital economy: Estimating the value of increased product variety at online booksellers. Management Science, 49(11), 1580-1596.

Casaburi, L., Glennerster, R., \& Suri, T. (2013). Rural roads and intermediated trade: Regression discontinuity evidence from Sierra Leone. Working Paper, University of Zurich.

Chapman, R., \& Slaymaker, T. (2002). ICTs and rural development: review of the literature, current interventions and opportunities for action. Food and Agriculture Organization (FAO) Report.

CNNIC. (2015a). The 36th statistical report on internet development in china. China Internet Network Information Center (CNNIC).

CNNIC. (2015b). Research report on china's online shopping market in 2014. China Internet Network Information Center (CNNIC).

Dolfen, P., Einav, L., Klenow, P. J., Klopack, B., Levin, J. D., Levin, L., \& Best, W. (2017). Assessing the gains from e-commerce. Working paper, Stanford University.

Fan, J., Tang, L., Zhu, W., \& Zou, B. (2016). The Alibaba effect: Spatial consumption inequality and the welfare gains from e-commerce. Working Paper, Michigan State University.

Forman, C., Goldfarb, A., \& Greenstein, S. (2012). The internet and local wages: A puzzle. American Economic Review, 102(1), 556-75.

FRED. (2016). E-commerce retail sales as a percent of total sales (ECOMPCTSA). Economic Data, Federal Reserve of St. Louis.

Goldmanis, M., Hortaçsu, A., Syverson, C., \& Emre, Ö. (2010). E-commerce and the market structure of retail industries. The Economic Journal, 120(545), 651-682.

Goyal, A. (2010). Information, direct access to farmers, and rural market performance in Central India. American Economic Journal: Applied Economics, 2(3), 22-45.

Hjort, J., \& Poulsen, J. (2018). The arrival of fast internet and skilled job creation in Africa. Forthcoming, American Economic Review.

Jensen, R. (2007). The digital provide: Information (technology), market performance, and welfare in the south indian fisheries sector. The Quarterly Journal of Economics, 122(3), 879-924.

Kling, J. R., Liebman, J. B., \& Katz, L. F. (2007). Experimental analysis of neighborhood effects. Econometrica, 75(1), 83-119. 
Luo, X., \& Niu, C. (2019). E-Commerce participation and household income growth in Taobao Villages. World Bank Poverty and Equity Global Practice Working Paper 198.

Luo, X., Wang, Y., \& Zhang, X. (2019). E-Commerce development and household consumption growth in China. World Bank Policy Research Working Paper 8810.

MCIT. (2016). Egypt's national e-commerce strategy. Ministry of Communications and Information Technology Report.

McKinsey. (2016). China's e-tail revolution. Report, McKinsey Global Institute.

MEITY. (2016). Digital india: Driving e-commerce in rural and semi urban India. Ministry of Electronics and IT Report.

Miguel, E., \& Kremer, M. (2004). Worms: Identifying impacts on education and health in the presence of treatment externalities. Econometrica, 72(1), 159-217.

PFSweb. (2016). China's e-commerce market 2015. Annual Market Report.

PM. (2016). Vietnam's e-commerce development masterplan. Vietnam's Office of the Prime Minister Report.

Statista. (2016). Online retail statistics for China. Market Statistics.

UNCTAD. (2016). E-commerce opens new opportunities for developing countries. UNCTAD/PRESS/U14/IN/2016/011.

Van de Walle, D. (2009). Impact evaluation of rural road projects. Journal of Development Effectiveness, 1(1), 15-36.

WB. (2016). World development report 2016: Digital dividends. 


\section{Figures and Tables}

Figure 1: Timeline of Adjustment: Village E-Commerce Consumption and Out-Shipments
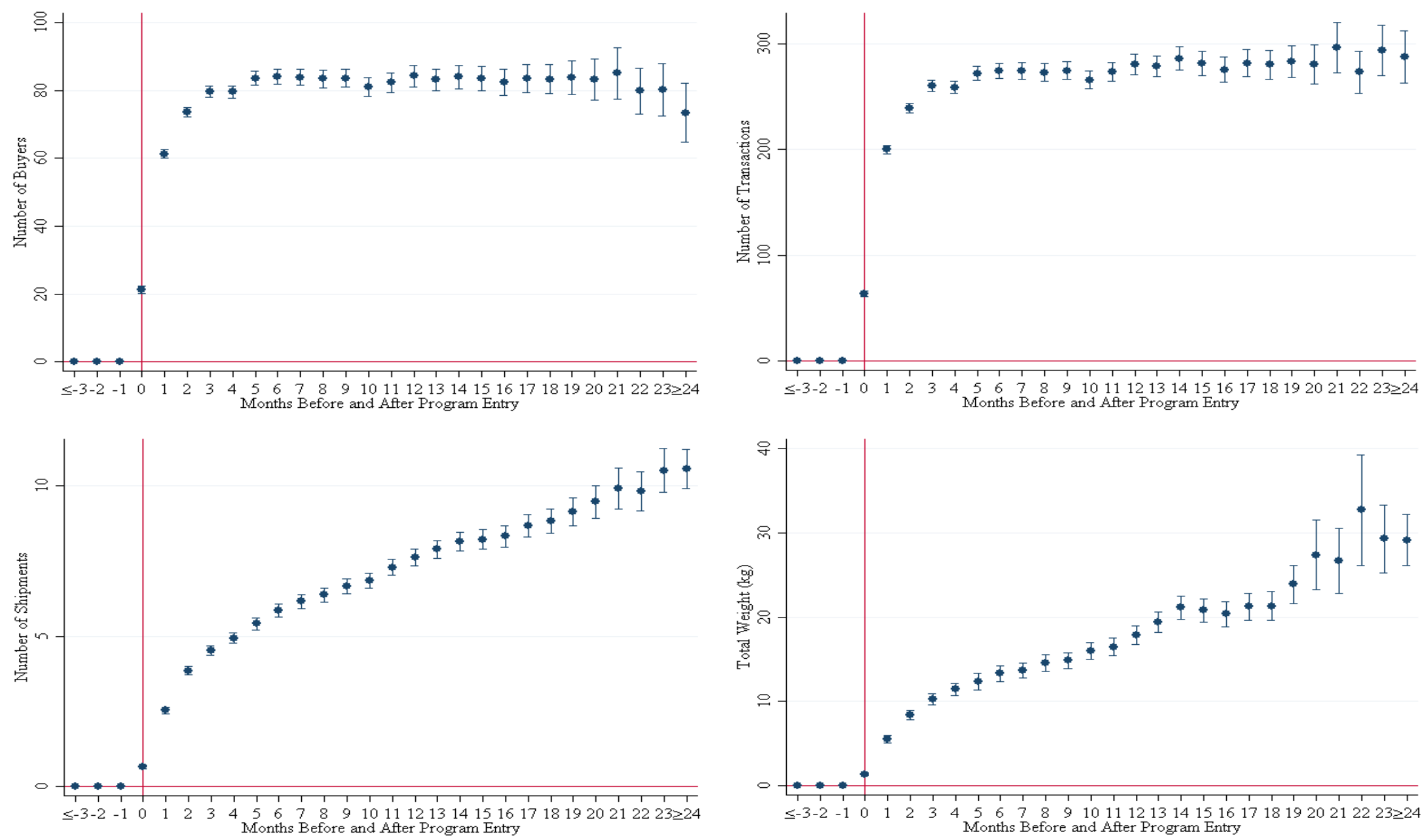

Notes: Figure shows point estimates from a regression of depicted outcomes on months since program entry and village and month fixed effects. Outcomes are the number of buyers (top left), the number of transactions (top right), the number of out-shipments (bottom left) and the total weight of out-shipments (bottom right) per village. The data are from the e-commerce firm's internal database and contain the universe of village purchase transactions from November 2015 to April 2017 and the universe of sales transations from January 2016 to April 2017 in the five provinces of Anhui, Guangxi, Guizhou, Henan, and Yunnan (roughly 11,900 villages in total). The last point estimate of each plot pools months 24 to 28. The figure shows 95 percent confidence intervals based on standard errors that are clustered at the level of villages. See Section 2.2 for discussion. 
Figure 2: Heterogeneity of Gains from E-Commerce
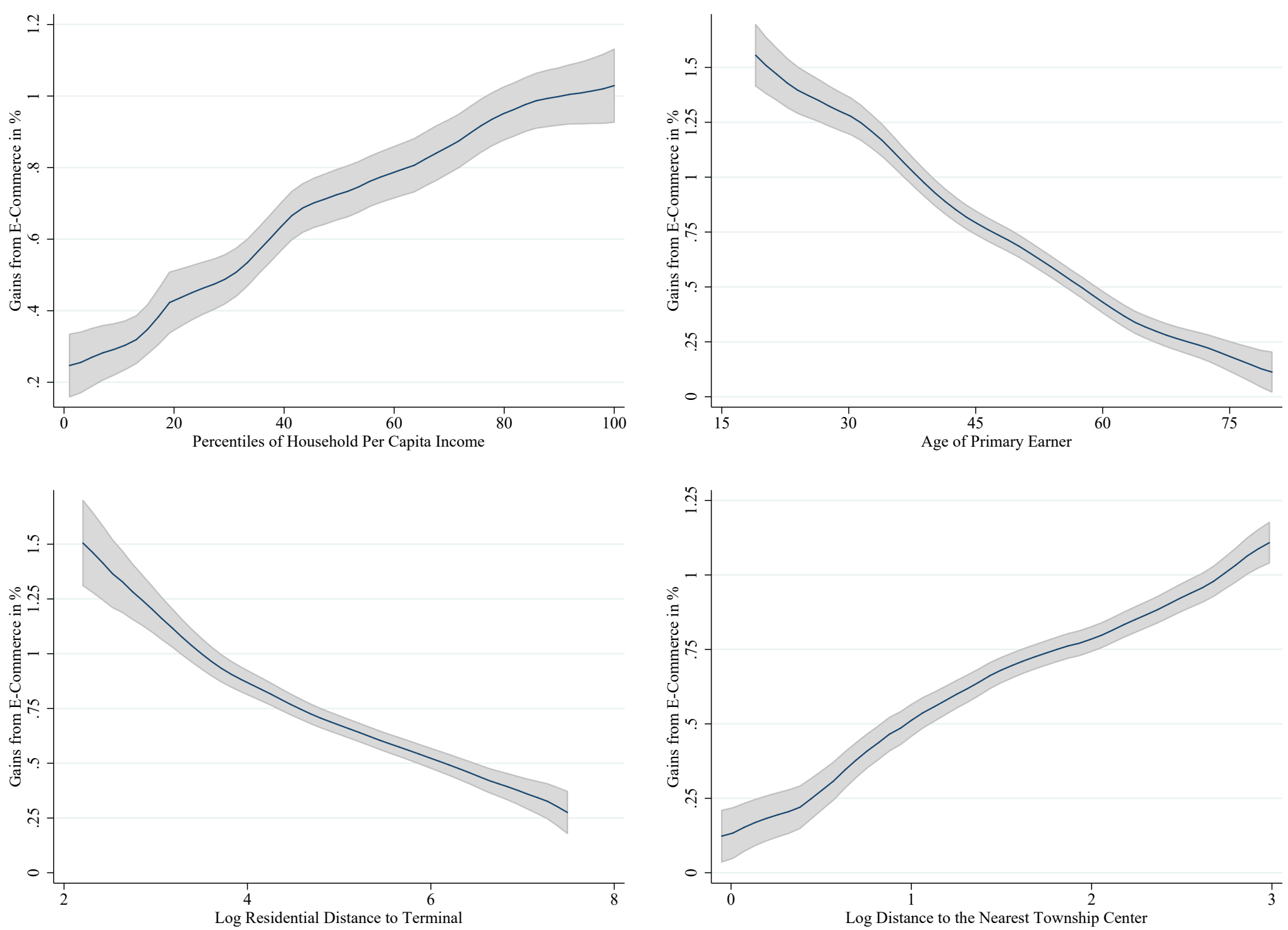

Notes: Figure shows predicted average gains (users and non-users) in terms of percentage point reductions in household retail cost of living as a function of household per capita income (top left), age of primary earner (top right), residential distance to terminal (bottom left), and distance to the nearest township center (bottom right). Predictions are based on treatment effects from bottom panel of Table 2. The figure depicts 95 percent confidence intervals that are based on clustering standard errors at the village level. See Section 3 for discussion. 
Table 1: Average Effects

\section{-- Panel A: Consumption --}

\begin{tabular}{|c|c|c|c|c|c|c|c|c|c|c|c|c|}
\hline & \multicolumn{2}{|c|}{$\begin{array}{l}\text { Monthly Retail } \\
\text { Expenditure Per } \\
\text { Capita in RMB }\end{array}$} & \multicolumn{2}{|c|}{$\begin{array}{l}\text { Has Bought Something } \\
\text { Through E-Comm } \\
\text { Option (Yes=1) }\end{array}$} & \multicolumn{2}{|c|}{$\begin{array}{c}\text { Share of E-Comm Option } \\
\text { in Monthly Total Retail } \\
\text { Expenditure }\end{array}$} & \multicolumn{2}{|c|}{$\begin{array}{l}\text { Share of E-Comm } \\
\text { Option in Monthly } \\
\text { Durables Expenditure }\end{array}$} & \multicolumn{2}{|c|}{$\begin{array}{c}\text { Share of E-Comm Option } \\
\text { in Monthly Non-Durables } \\
\text { Expenditure }\end{array}$} & \multicolumn{2}{|c|}{$\begin{array}{l}\text { Consumption } \\
\text { Effects } \\
\text { (K-L-K Index) }\end{array}$} \\
\hline & ITT & TOT & ITT & TOT & ITT & TOT & ITT & TOT & ITT & TOT & ITT & TOT \\
\hline Treat & $\begin{array}{l}-22.09 \\
(31.99)\end{array}$ & $\begin{array}{l}-41.20 \\
(60.22)\end{array}$ & $\begin{array}{c}0.0484^{\mathrm{a}} \\
(0.0167)\end{array}$ & $\begin{array}{c}0.0894^{\mathrm{a}} \\
(0.0268)\end{array}$ & $\begin{array}{l}0.00668^{\mathbf{a}} \\
(0.00239)\end{array}$ & $\begin{array}{c}0.0124^{\mathrm{a}} \\
(0.00435)\end{array}$ & $\begin{array}{c}0.0408^{\mathbf{b}} \\
(0.0160)\end{array}$ & $\begin{array}{c}0.0686^{\mathrm{a}} \\
(0.0263)\end{array}$ & $\begin{array}{c}0.00538^{\mathrm{a}} \\
(0.00196)\end{array}$ & $\begin{array}{c}0.01^{\mathrm{a}} \\
(0.00356)\end{array}$ & $\begin{array}{c}0.478^{\mathrm{a}} \\
(0.0336)\end{array}$ & $\begin{array}{c}0.885^{\mathrm{a}} \\
(0.126)\end{array}$ \\
\hline R-Squared & 0.038 & & 0.008 & & 0.006 & & 0.012 & & 0.003 & & 0.118 & \\
\hline Control Mean & \multicolumn{2}{|c|}{592.21} & \multicolumn{2}{|c|}{0.0501} & \multicolumn{2}{|c|}{0.00277} & \multicolumn{2}{|c|}{0.0152} & \multicolumn{2}{|c|}{0.0027} & \multicolumn{2}{|c|}{0.00} \\
\hline 1st Stage F & & 43.92 & & 45.56 & & 44.03 & & 52.43 & & 44.11 & & 44.94 \\
\hline \multirow[t]{4}{*}{ Obs } & 3,436 & 3,436 & 3,518 & 3,518 & 3,434 & 3,434 & 768 & 768 & 3,433 & 3,433 & 3,539 & 3,539 \\
\hline & \multicolumn{4}{|c|}{ - Panel B: Nominal Incomes -- } & \multicolumn{8}{|c|}{-- Panel C: Local Retail Prices -- } \\
\hline & \multicolumn{2}{|c|}{$\begin{array}{c}\text { Monthly Income } \\
\text { Per Capita in } \\
\text { RMB }\end{array}$} & \multicolumn{2}{|c|}{$\begin{array}{l}\text { Income Effects } \\
\text { (K-L-K Index) }\end{array}$} & \multicolumn{2}{|c|}{ Log Prices } & \multicolumn{2}{|c|}{$\begin{array}{l}\text { Product Replacement } \\
\text { Dummy }\end{array}$} & \multicolumn{2}{|c|}{$\begin{array}{l}\text { Production Addition } \\
\text { Dummy }\end{array}$} & \multicolumn{2}{|c|}{$\begin{array}{l}\text { Price Effects } \\
\text { (K-L-K Index) }\end{array}$} \\
\hline & ITT & TOT & ITT & TOT & ITT & TOT & ITT & TOT & ITT & TOT & ITT & TOT \\
\hline Treat & $\begin{array}{l}-7.864 \\
(70.78)\end{array}$ & $\begin{array}{l}-14.53 \\
(129.9)\end{array}$ & $\begin{array}{l}-0.0309 \\
(0.0349)\end{array}$ & $\begin{array}{l}-0.0572 \\
(0.0646)\end{array}$ & $\begin{array}{c}0.0189 \\
(0.0142)\end{array}$ & $\begin{array}{c}0.0352 \\
(0.0263)\end{array}$ & $\begin{array}{l}-0.00392 \\
(0.0300)\end{array}$ & $\begin{array}{l}-0.00747 \\
(0.0569)\end{array}$ & $\begin{array}{l}2.194^{\mathbf{b}} \\
(1.073)\end{array}$ & $\begin{array}{l}4.020^{\mathrm{c}} \\
(2.278)\end{array}$ & $\begin{array}{l}-0.217 \\
(0.134)\end{array}$ & $\begin{array}{l}-0.389 \\
(0.260)\end{array}$ \\
\hline R-Squared & 0.038 & & 0.002 & & 0.893 & & 0.00 & & 0.277 & & 0.010 & \\
\hline Control Mean & 915 & 5.51 & & & 1. & & & & & & 0.0 & \\
\hline 1st Stage F & & 45.33 & & 45.01 & & 41.66 & & 39.82 & & 19.69 & & 24.05 \\
\hline Obs & 3,437 & 3,437 & 3,538 & 3,538 & 6,877 & 6,877 & 8,956 & 8,956 & 312 & 312 & 343 & 343 \\
\hline
\end{tabular}

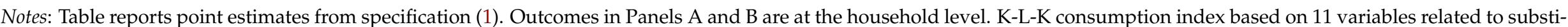

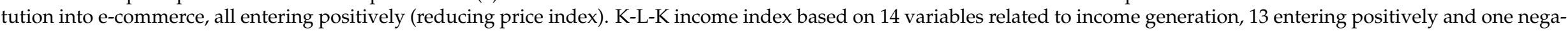

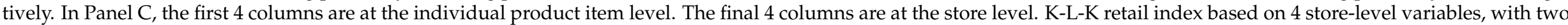

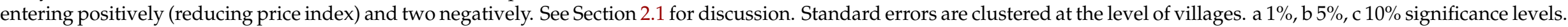


Table 2: Heterogeneity Across Households and Villages

\begin{tabular}{|c|c|c|c|c|c|c|c|}
\hline \multirow[t]{2}{*}{$\begin{array}{l}\text { Type of } \\
\text { Heterogeneity }\end{array}$} & & \multicolumn{2}{|c|}{$\begin{array}{l}\text { Household Has Bought Something } \\
\text { through E-Comm Option (Yes=1) }\end{array}$} & \multicolumn{2}{|c|}{$\begin{array}{l}\text { Monthly Income Per } \\
\text { Capita in RMB }\end{array}$} & \multicolumn{2}{|c|}{$\begin{array}{l}\text { Log Local Retail } \\
\text { Prices }\end{array}$} \\
\hline & & ITT & TOT & ITT & TOT & $\begin{array}{l}\text { ITT } \\
\end{array}$ & TOT \\
\hline $\begin{array}{l}\text { Village Was } \\
\text { Previously }\end{array}$ & Treat & $\begin{array}{l}0.0578^{\mathrm{a}} \\
(0.0188)\end{array}$ & $\begin{array}{c}0.106^{\mathrm{a}} \\
(0.0283)\end{array}$ & $\begin{array}{l}-15.00 \\
(77.55)\end{array}$ & $\begin{array}{l}-27.15 \\
(140.1)\end{array}$ & $\begin{array}{c}0.0114 \\
(0.0144)\end{array}$ & $\begin{array}{c}0.0215 \\
(0.0273)\end{array}$ \\
\hline $\begin{array}{l}\text { Connected to } \\
\text { Parcel Delivery } \\
(\mathrm{Yes}=1)\end{array}$ & $\begin{array}{l}\text { Treat* } \\
\text { Delivery } \\
\text { First Stage F }\end{array}$ & $\begin{array}{l}-0.0606^{\mathbf{b}} \\
(0.0253)\end{array}$ & $\begin{array}{c}-0.111^{\mathbf{b}} \\
(0.0443) \\
2.682\end{array}$ & $\begin{array}{c}50.17 \\
(171.1)\end{array}$ & $\begin{array}{c}96.91 \\
(339.0) \\
2.694\end{array}$ & $\begin{array}{c}0.0417 \\
(0.0377)\end{array}$ & $\begin{array}{c}0.0739 \\
(0.0572) \\
17.26\end{array}$ \\
\hline Village & Treat & $\begin{array}{l}-0.0144 \\
(0.0281)\end{array}$ & $\begin{array}{l}-0.00652 \\
(0.0411)\end{array}$ & $\begin{array}{l}-23.61 \\
(181.7)\end{array}$ & $\begin{array}{l}-43.80 \\
(289.1)\end{array}$ & $\begin{array}{l}-0.0219 \\
(0.0375)\end{array}$ & $\begin{array}{l}-0.0322 \\
(0.0632)\end{array}$ \\
\hline $\begin{array}{l}\text { Distance to } \\
\text { Township } \\
\text { Center }\end{array}$ & $\begin{array}{l}\text { Treat * Log } \\
\text { Dist Township } \\
\text { First Stage F }\end{array}$ & $\begin{array}{c}0.0384^{\mathbf{b}} \\
(0.0161)\end{array}$ & $\begin{array}{c}0.0606^{\mathrm{a}} \\
(0.0223) \\
15.55 \\
\end{array}$ & $\begin{array}{c}0.422 \\
(97.49)\end{array}$ & $\begin{array}{c}0.422 \\
(152.0) \\
15.66 \\
\end{array}$ & $\begin{array}{c}0.0216 \\
(0.0198)\end{array}$ & $\begin{array}{c}0.0358 \\
(0.0336) \\
16.96 \\
\end{array}$ \\
\hline & Treat & $\begin{array}{c}0.141^{\mathrm{a}} \\
(0.0505)\end{array}$ & $\begin{array}{c}0.223^{\mathrm{a}} \\
(0.0777)\end{array}$ & $\begin{array}{l}-136.5 \\
(172.5)\end{array}$ & $\begin{array}{l}-238.0 \\
(286.5)\end{array}$ & & \\
\hline $\begin{array}{l}\text { Primary } \\
\text { Earner's Age }\end{array}$ & Treat* Age & $\begin{array}{c}-0.00172^{b} \\
(0.000773)\end{array}$ & $\begin{array}{l}-0.00251^{\mathrm{c}} \\
(0.00129)\end{array}$ & $\begin{array}{c}2.563 \\
(2.734)\end{array}$ & $\begin{array}{c}4.554 \\
(4.825)\end{array}$ & & \\
\hline & First Stage F & & 16.04 & & 16.34 & & \\
\hline $\begin{array}{l}\text { Primary } \\
\text { Earner's } \\
\text { Educaction }\end{array}$ & $\begin{array}{l}\text { Treat * Years } \\
\text { of Education } \\
\text { First Stage F }\end{array}$ & $\begin{array}{c}0.0408^{\mathrm{c}} \\
(0.0206) \\
0.00164 \\
(0.00266)\end{array}$ & $\begin{array}{c}0.0979^{\mathbf{b}} \\
(0.0412) \\
-0.000432 \\
(0.00504) \\
8.456 \\
\end{array}$ & $\begin{array}{l}52.81 \\
(83.52) \\
-8.672 \\
(12.14)\end{array}$ & $\begin{array}{c}119.7 \\
(195.0) \\
-17.80 \\
(24.03) \\
8.662 \\
\end{array}$ & & \\
\hline Household & Treat & $\begin{array}{l}0.00863 \\
(0.0214)\end{array}$ & $\begin{array}{c}0.0220 \\
(0.0375)\end{array}$ & $\begin{array}{l}35.83 \\
(96.84)\end{array}$ & $\begin{array}{l}59.45 \\
(165.5)\end{array}$ & & \\
\hline $\begin{array}{l}\text { Income Per } \\
\text { Capita }\end{array}$ & $\begin{array}{l}\text { Treat* Log } \\
\text { Income } \\
\text { First Stage F } \\
\end{array}$ & $\begin{array}{c}0.00708^{\mathbf{b}} \\
(0.00327)\end{array}$ & $\begin{array}{c}0.0120^{\mathbf{b}} \\
(0.00544) \\
22.67 \\
\end{array}$ & $\begin{array}{c}-9.201 \\
(21.22)\end{array}$ & $\begin{array}{c}-15.78 \\
(36.32) \\
22.57 \\
\end{array}$ & & \\
\hline $\begin{array}{l}\text { Household } \\
\text { Distance to } \\
\text { Planned } \\
\text { Terminal }\end{array}$ & $\begin{array}{l}\text { Treat * Log } \\
\text { Dist Terminal } \\
\text { First Stage F }\end{array}$ & $\begin{array}{c}0.142^{\mathbf{b}} \\
(0.0600) \\
-0.0177^{\mathrm{c}} \\
(0.0100)\end{array}$ & $\begin{array}{c}0.227^{\mathbf{b}} \\
(0.110) \\
-0.0264 \\
(0.0196) \\
9.899 \\
\end{array}$ & $\begin{array}{l}185.8 \\
(350.6) \\
-36.53 \\
(61.53)\end{array}$ & $\begin{array}{c}400.0 \\
(697.5) \\
-79.65 \\
(128.5) \\
9.325 \\
\end{array}$ & & \\
\hline & $\begin{array}{l}\text { Treat } \\
\text { Treat* } \\
\text { Delivery }\end{array}$ & $\begin{array}{c}0.153^{\mathrm{c}} \\
(0.0811) \\
-0.0401 \\
(0.0286)\end{array}$ & $\begin{array}{c}0.287^{\mathbf{b}} \\
(0.141) \\
-0.106 \\
(0.0690)\end{array}$ & $\begin{array}{c}174.5 \\
(329.9) \\
102.1 \\
(121.1)\end{array}$ & $\begin{array}{c}330.1 \\
(612.1) \\
253.3 \\
(308.1)\end{array}$ & $\begin{array}{c}-0.0398 \\
(0.0362) \\
0.0413 \\
(0.0361)\end{array}$ & $\begin{array}{c}-0.0435 \\
(0.0531) \\
0.0517 \\
(0.0622)\end{array}$ \\
\hline & $\begin{array}{l}\text { Treat } * \text { Log } \\
\text { Dist Township }\end{array}$ & $\begin{array}{l}0.0457^{\mathrm{a}} \\
(0.0173)\end{array}$ & $\begin{array}{l}0.0809^{\mathrm{a}} \\
(0.0296)\end{array}$ & $\begin{array}{l}-42.86 \\
(58.39)\end{array}$ & $\begin{array}{l}-93.17 \\
(128.5)\end{array}$ & $\begin{array}{c}0.0284 \\
(0.0188)\end{array}$ & $\begin{array}{c}0.0380 \\
(0.0312)\end{array}$ \\
\hline Combined & Treat* Age & $\begin{array}{l}-0.00181^{\mathbf{b}} \\
(0.000775)\end{array}$ & $\begin{array}{l}-0.00314^{b} \\
(0.00130)\end{array}$ & $\begin{array}{c}0.587 \\
(2.555)\end{array}$ & $\begin{array}{l}1.266 \\
(4.602)\end{array}$ & & \\
\hline & $\begin{array}{l}\text { Treat* Years } \\
\text { of Education } \\
\text { Treat* Log } \\
\text { Income } \\
\text { Treat* Log } \\
\text { Dist Terminal } \\
\text { First Stage F }\end{array}$ & $\begin{array}{c}0.000384 \\
(0.00267) \\
0.00907^{\mathbf{a}} \\
(0.00339) \\
-0.0248^{\mathbf{b}} \\
(0.0109)\end{array}$ & $\begin{array}{c}-0.00377 \\
(0.00497) \\
0.0162^{\mathrm{a}} \\
(0.00556) \\
-0.0411^{\mathbf{c}} \\
(0.0222) \\
0.479\end{array}$ & $\begin{array}{l}-2.230 \\
(10.01) \\
-8.451 \\
(22.00) \\
-16.48 \\
(45.01)\end{array}$ & $\begin{array}{c}-1.954 \\
(21.43) \\
-14.28 \\
(37.97) \\
-34.37 \\
(94.93) \\
0.419\end{array}$ & & \\
\hline
\end{tabular}

Notes: Based on the same samples as Table 1. See Section 2.1 for discussion. Standard errors are clustered at the level of villages. a $1 \%, \mathrm{~b}$ $5 \%$, c $10 \%$ significance levels. 
Table 3: Average Effects On Household Welfare

\begin{tabular}{lccc|ccc}
\hline & \multicolumn{2}{c|}{ Unweighted (Effects in Sample) } & \multicolumn{2}{c}{ Weighted (Effects in Village Population) } \\
\hline & $\begin{array}{c}\text { Durables } \\
\text { Consumption }\end{array}$ & $\begin{array}{c}\text { Non-Durables } \\
\text { Consumption }\end{array}$ & $\begin{array}{c}\text { Total Retail } \\
\text { Consumption }\end{array}$ & $\begin{array}{c}\text { Durables } \\
\text { Consumption }\end{array}$ & $\begin{array}{c}\text { Non-Durables } \\
\text { Consumption }\end{array}$ & $\begin{array}{c}\text { Total Retail } \\
\text { Consumptio }\end{array}$ \\
\hline Reduction in Retail Cost of & $\begin{array}{c}3.379 \% \\
(0.03)\end{array}$ & $\begin{array}{c}0.481 \% \\
(0.003)\end{array}$ & $\begin{array}{c}0.824 \% \\
(0.005)\end{array}$ & $\begin{array}{c}2.962 \% \\
(0.03)\end{array}$ & $\begin{array}{c}0.429 \% \\
(0.003)\end{array}$ & $\begin{array}{c}0.73 \% \\
(0.005)\end{array}$ \\
\hline Living for All Households & $19.884 \%$ & $3.806 \%$ & $5.597 \%$ & $16.637 \%$ & $3.217 \%$ & $4.722 \%$ \\
Reduction in Retail Cost of & $(0.221)$ & $(0.028)$ & $(0.034)$ & $(0.224)$ & $(0.025)$ & $(0.032)$ \\
\hline Living Among Users & & &
\end{tabular}

Notes: Table reports average household gains in terms of percentage point reductions in retail cost of living for different consumption categories and groups of households. Estimates are based on equation (3) using treatment effects on household substitution into the new e-commerce option. The left panel reports unweighted results, and the right panel adjusts the weight of each household using sampling weights. Standard errors are bootstrapped across 1000 iterations, taking into account that the treatment effects are point estimates. See Section 3 for discussion. 


\section{Appendix}

Appenix A presents additional figures and tables. Appendix B describes the construction of the K-L-K outcome indices. Appendix C presents additional analysis on the role of GE spillovers. Appendix D provides additional estimation results using the firm's admin database. Appendix E provides details on the welfare analysis. Appendix F presents details on the program, experimental design, field staff training, quality management and data.

\section{Appendix A: Additional Figures and Tables}

Figure A.1: Provinces and Counties Where RCT Was Implemented

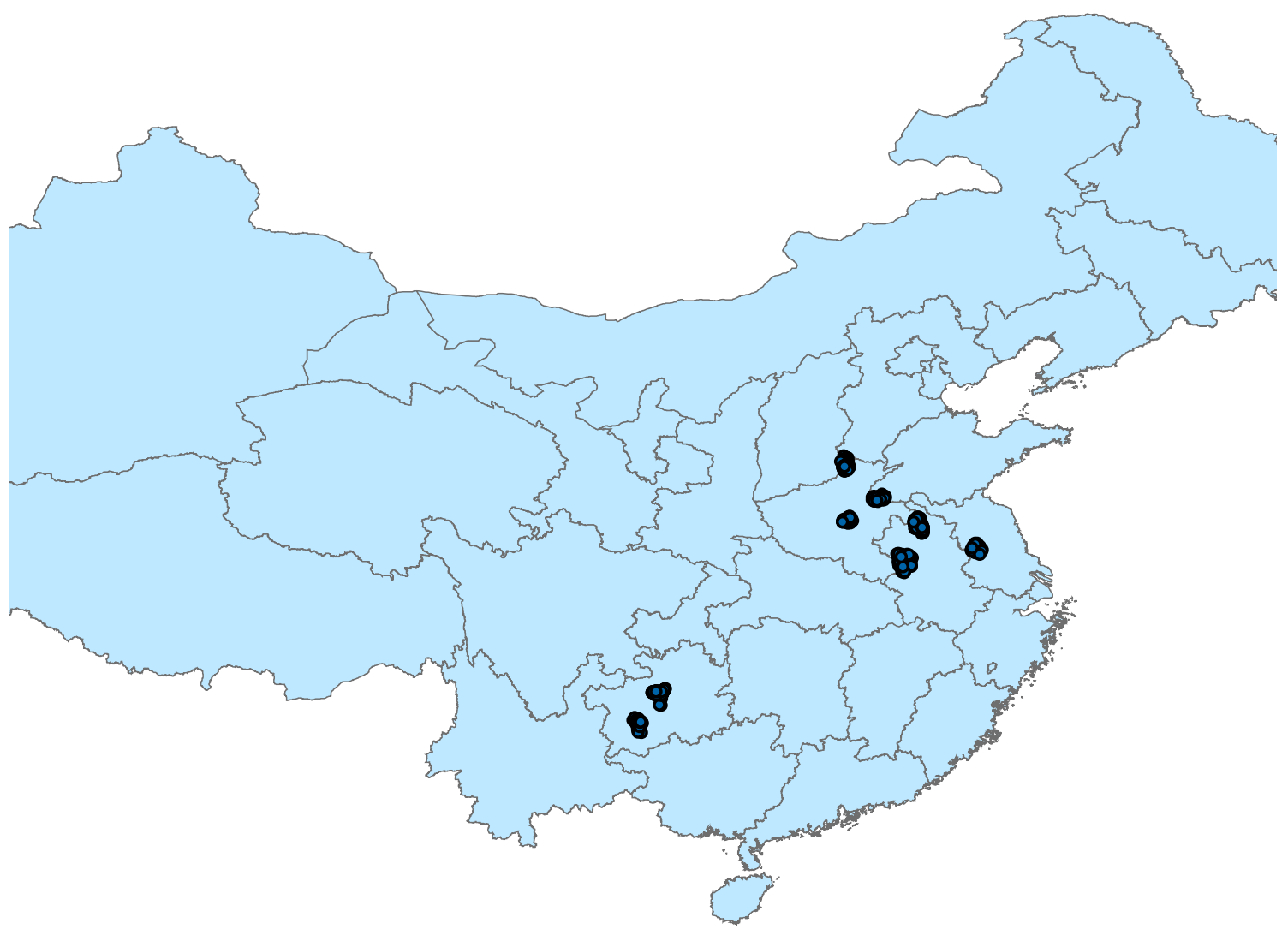

Notes: Map shows the location of our eight RCT counties in the three provinces of Anhui, Guizhou and Henan. The dots indicate participating villages and the boundaries indicate Mainland Chinese provinces. Section Design/Data Section and Appendix F for discussion. 
Table A.1: Descriptive Statistics: Individual Level

\begin{tabular}{|c|c|c|c|c|c|c|}
\hline & & $\begin{array}{l}\text { Full Sample at } \\
\text { Baseline }\end{array}$ & $\begin{array}{c}\text { Treatment } \\
\text { Villages at } \\
\text { Baseline } \\
\end{array}$ & $\begin{array}{l}\text { Control Villages } \\
\text { at Baseline }\end{array}$ & $\begin{array}{c}\text { P-Value } \\
(\text { Treat-Control }=0)\end{array}$ & $\begin{array}{l}\text { Control Villages } \\
\text { at Endline }\end{array}$ \\
\hline \multirow{4}{*}{ Age } & Median & 44.000 & 44.000 & 43.000 & \multirow{4}{*}{0.208} & 46.000 \\
\hline & Mean & 38.950 & 39.329 & 38.407 & & 39.943 \\
\hline & Standard Deviation & 23.580 & 23.658 & 23.460 & & 23.759 \\
\hline & Number of Obs & 8491 & 5001 & 3490 & & 4194 \\
\hline \multirow{4}{*}{ Gender $($ Female $=1)$} & Median & 1.000 & 1.000 & 1.000 & \multirow{4}{*}{0.025} & 1.000 \\
\hline & Mean & 0.534 & 0.526 & 0.546 & & 0.537 \\
\hline & Standard Deviation & 0.499 & 0.499 & 0.498 & & 0.499 \\
\hline & Number of Obs & 8484 & 5001 & 3483 & & 4188 \\
\hline \multirow{4}{*}{$\begin{array}{l}\text { Employed (for age }>15) \\
(Y e s=1)\end{array}$} & Median & 1.000 & 1.000 & 1.000 & \multirow{4}{*}{0.882} & 1.000 \\
\hline & Mean & 0.767 & 0.766 & 0.769 & & 0.762 \\
\hline & Standard Deviation & 0.423 & 0.424 & 0.422 & & 0.426 \\
\hline & Number of Obs & 6070 & 3590 & 2480 & & 3015 \\
\hline \multirow{4}{*}{$\begin{array}{l}\text { Farmer (for age }>15) \\
(Y e s=1)\end{array}$} & Median & 1.000 & 1.000 & 1.000 & \multirow{4}{*}{0.971} & 1.000 \\
\hline & Mean & 0.527 & 0.527 & 0.526 & & 0.513 \\
\hline & Standard Deviation & 0.499 & 0.499 & 0.499 & & 0.500 \\
\hline & Number of Obs & 6369 & 3760 & 2609 & & 3144 \\
\hline \multirow{4}{*}{$\begin{array}{l}\text { No Schooling (for } \\
\text { age }>15) \text { (No } \\
\text { School }=1)\end{array}$} & Median & 0.000 & 0.000 & 0.000 & \multirow{4}{*}{0.745} & 0.000 \\
\hline & Mean & 0.270 & 0.273 & 0.266 & & 0.319 \\
\hline & Standard Deviation & 0.444 & 0.446 & 0.442 & & 0.466 \\
\hline & Number of Obs & 6368 & 3758 & 2610 & & 3132 \\
\hline \multirow{4}{*}{$\begin{array}{l}\text { Completed Junior High } \\
\text { School (for age }>15) \\
(Y e s=1)\end{array}$} & Median & 0.000 & 0.000 & 0.000 & \multirow{4}{*}{0.419} & 0.000 \\
\hline & Mean & 0.437 & 0.429 & 0.449 & & 0.422 \\
\hline & Standard Deviation & 0.496 & 0.495 & 0.498 & & 0.494 \\
\hline & Number of Obs & 6368 & 3758 & 2610 & & 3132 \\
\hline \multirow{4}{*}{$\begin{array}{l}\text { Completed Senior } \\
\text { High School (for } \\
\text { age }>18)(Y e s=1)\end{array}$} & Median & 0.000 & 0.000 & 0.000 & \multirow{4}{*}{0.969} & 0.000 \\
\hline & Mean & 0.104 & 0.104 & 0.104 & & 0.097 \\
\hline & Standard Deviation & 0.305 & 0.305 & 0.305 & & 0.296 \\
\hline & Number of Obs & 6286 & 3719 & 2567 & & 3096 \\
\hline
\end{tabular}

Notes: See Design/Data Section and Appendix F for discussion. 
Table A.2: Descriptive Statistics: Household Level

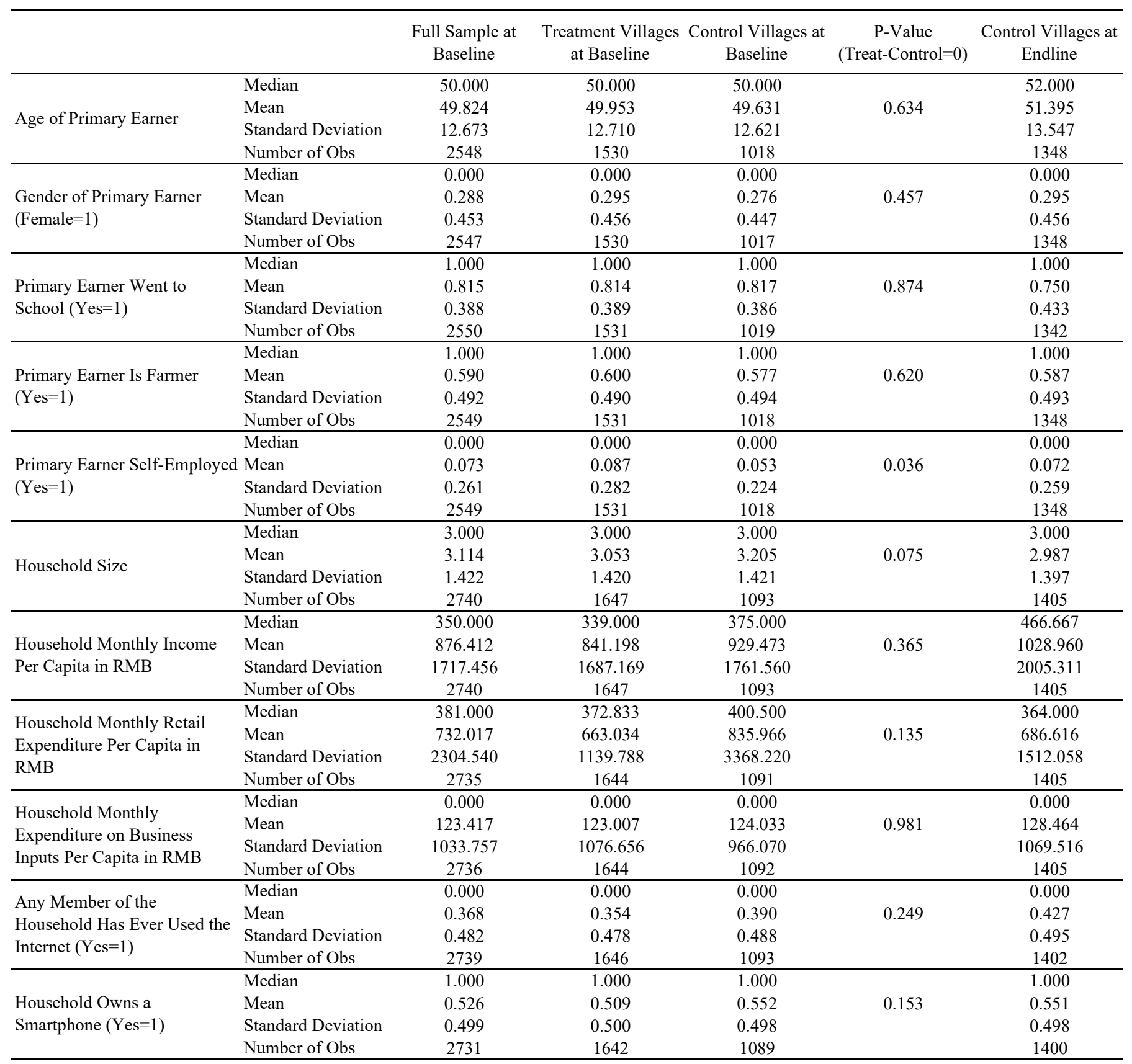

Notes: See Design/Data Section and Appendix F for discussion. 
Table A.3: Descriptive Statistics: Household Level - Continued

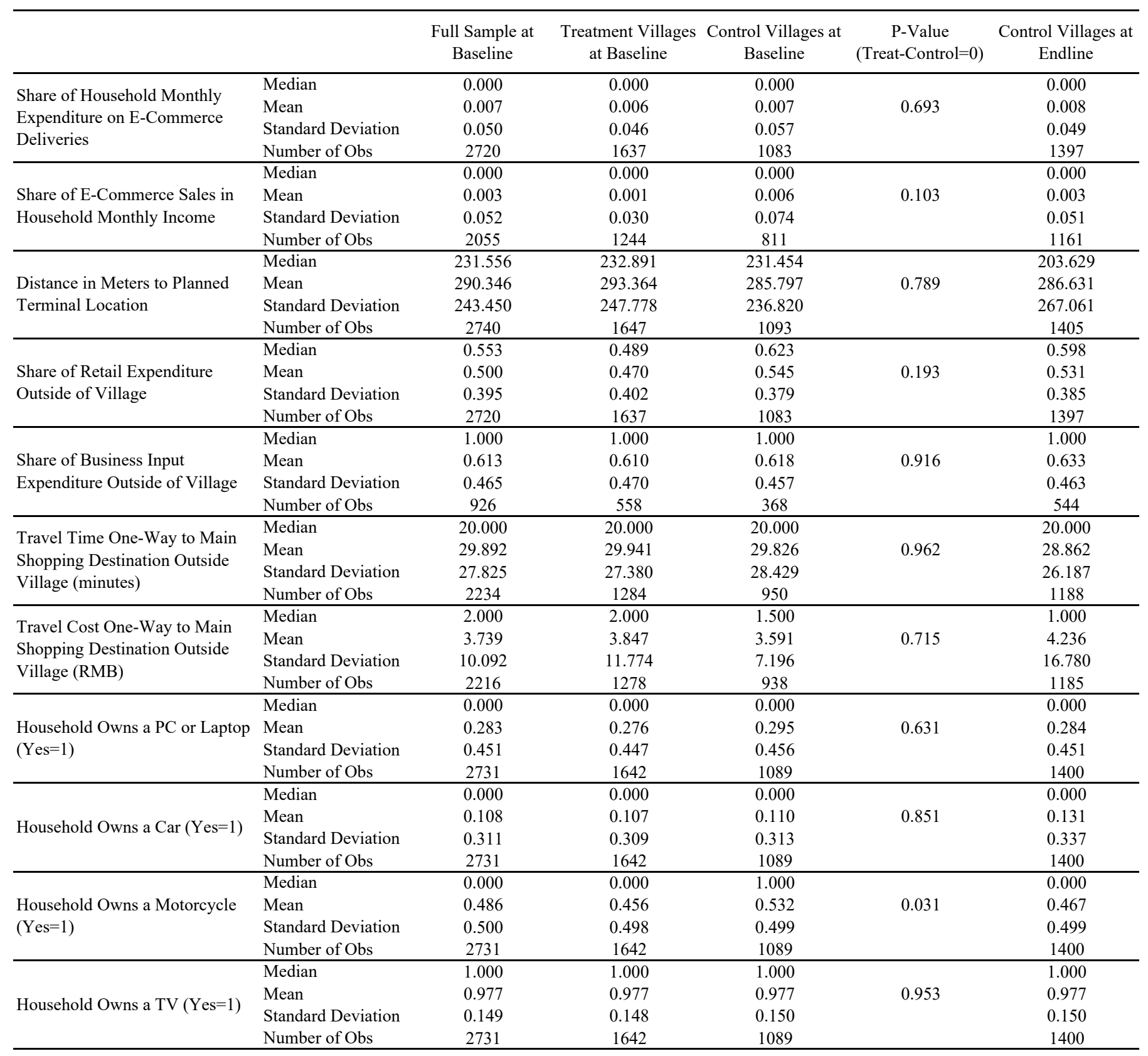

Notes: See Design/Data Section and Appendix F for discussion. 
Table A.4: Descriptive Statistics: Local Retail Prices

\begin{tabular}{|c|c|c|c|c|c|c|}
\hline & & $\begin{array}{l}\text { Full Sample at } \\
\text { Baseline }\end{array}$ & $\begin{array}{c}\text { Treatment Villages } \\
\text { at Baseline }\end{array}$ & $\begin{array}{c}\text { Control Villages at } \\
\text { Baseline }\end{array}$ & $\begin{array}{c}\text { P-Value } \\
(\text { Treat-Control }=0)\end{array}$ & $\begin{array}{l}\text { Control Villages at } \\
\text { Endline }\end{array}$ \\
\hline \multirow{4}{*}{$\begin{array}{l}\text { Number of Stores at Village } \\
\text { Level }\end{array}$} & Median & 3.00 & 3.00 & 2.00 & \multirow{4}{*}{0.33} & 2.00 \\
\hline & Mean & 4.15 & 4.38 & 3.79 & & 3.61 \\
\hline & Standard Deviation & 2.94 & 2.91 & 2.98 & & 2.99 \\
\hline & Number of Obs & 99 & 60 & 39 & & 38 \\
\hline \multirow{3}{*}{$\begin{array}{l}\text { Establishment Space in } \\
\text { Square Meters }\end{array}$} & Median & 50.00 & 50.00 & 40.00 & \multirow{3}{*}{0.35} & 50.00 \\
\hline & Standard Deviation & 320.38 & 89.60 & 532.73 & & 375.35 \\
\hline & Number of Obs & 361 & 238 & 123 & & 126 \\
\hline \multirow{4}{*}{$\begin{array}{l}\text { Number of Establishment's } \\
\text { New Products Added Over } \\
\text { Last Month }\end{array}$} & Median & 0.00 & 0.00 & 0.00 & \multirow{4}{*}{0.57} & 0.00 \\
\hline & Mean & 1.43 & 1.56 & 1.17 & & 0.63 \\
\hline & Standard Deviation & 7.44 & 8.88 & 3.42 & & 2.26 \\
\hline & Number of Obs & 330 & 215 & 115 & & 126 \\
\hline \multirow{3}{*}{$\begin{array}{l}\text { Prices of All Retail } \\
\text { Consumption ( } 9 \text { Product } \\
\text { Groups) in RMB }\end{array}$} & Median & 7.00 & 7.00 & 6.00 & \multirow{3}{*}{0.47} & 6.00 \\
\hline & Standard Deviation & 411.24 & 433.67 & 370.33 & & 390.31 \\
\hline & Number of Obs & 9382 & 5884 & 3498 & & 3259 \\
\hline \multirow{4}{*}{$\begin{array}{l}\text { Price Was Not Displayed on } \\
\text { Label (Needed to Ask }=1 \text { ) }\end{array}$} & Median & 1.00 & 1.00 & 1.00 & \multirow{4}{*}{0.97} & 1.00 \\
\hline & Mean & 0.67 & 0.66 & 0.67 & & 0.73 \\
\hline & Standard Deviation & 0.47 & 0.47 & 0.47 & & 0.44 \\
\hline & Number of Obs & 8977 & 5597 & 3380 & & 3370 \\
\hline \multirow{4}{*}{$\begin{array}{l}\text { Prices of Business or } \\
\text { Production Input in RMB }\end{array}$} & Median & 10.00 & 10.00 & 8.80 & \multirow{4}{*}{0.76} & 9.00 \\
\hline & Mean & 45.63 & 42.88 & 49.78 & & 43.84 \\
\hline & Standard Deviation & 195.09 & 206.23 & 177.46 & & 97.92 \\
\hline & Number of Obs & 444 & 267 & 177 & & 111 \\
\hline \multirow{4}{*}{$\begin{array}{l}\text { (1) Prices of Food and } \\
\text { Beverages in RMB }\end{array}$} & Median & 4.38 & 4.60 & 4.00 & \multirow{4}{*}{0.73} & 4.00 \\
\hline & Mean & 11.58 & 11.81 & 11.21 & & 10.05 \\
\hline & Standard Deviation & 24.35 & 23.31 & 25.99 & & 17.75 \\
\hline & Number of Obs & 4853 & 3021 & 1832 & & 1834 \\
\hline \multirow{2}{*}{$\begin{array}{l}\text { (2) Prices of Tobacco and } \\
\text { Alcohol in RMB }\end{array}$} & Median & 12.00 & 13.00 & 12.00 & & 13.00 \\
\hline & Number of Obs & 1331 & 818 & 513 & & 531 \\
\hline & Median & 10.00 & 10.00 & 9.98 & & 8.40 \\
\hline (3) Prices of Medicine and & Mean & 26.13 & 24.40 & 29.31 & 0.66 & 18.50 \\
\hline Health Products in RMB & Standard Deviation & 43.35 & 38.46 & 51.11 & & 33.77 \\
\hline & Number of Obs & 399 & 258 & 141 & & 90 \\
\hline & Median & 15.00 & 12.00 & 20.00 & & 22.00 \\
\hline (4) Prices of Clothing and & Mean & 46.31 & 45.69 & 47.79 & 0.90 & 57.00 \\
\hline Accessories in RMB & Standard Deviation & 74.71 & 71.49 & 82.13 & & 85.66 \\
\hline & Number of Obs & 401 & 282 & 119 & & 65 \\
\hline & Median & 10.00 & 10.00 & 9.00 & & 9.00 \\
\hline (5) Prices of Other Everyday & Mean & 14.68 & 14.53 & 14.93 & 0.93 & 13.10 \\
\hline Products in RMB & Standard Deviation & 31.03 & 32.69 & 28.06 & & 18.17 \\
\hline & Number of Obs & 1462 & 916 & 546 & & 626 \\
\hline & Median & 5.00 & 5.00 & 5.00 & & 5.83 \\
\hline (6) Prices of Fuel and Gas in & Mean & 11.65 & 15.36 & 8.08 & 0.26 & 5.82 \\
\hline RMB & Standard Deviation & 21.46 & 28.88 & 9.59 & & 0.23 \\
\hline & Number of Obs & 53 & 26 & 27 & & 4 \\
\hline & Median & 110.00 & 85.00 & 187.00 & & 398.00 \\
\hline (7) Prices of Furniture and & Mean & 1009.49 & 1001.66 & 1026.34 & 0.95 & 1167.30 \\
\hline Appliances in RMB & Standard Deviation & 1504.81 & 1583.03 & 1333.52 & & 1350.70 \\
\hline & Number of Obs & 183 & 125 & 58 & & 43 \\
\hline & Median & 449.00 & 609.50 & 17.50 & & 1799.00 \\
\hline (8) Prices of Electronics in & Mean & 917.05 & 976.41 & 782.14 & 0.59 & 1782.71 \\
\hline RMB & Standard Deviation & 1224.37 & 1242.82 & 1184.20 & & 871.58 \\
\hline & Number of Obs & 144 & 100 & 44 & & 45 \\
\hline & Median & 1440.00 & 1980.00 & 30.00 & & 2800.00 \\
\hline (9) Prices of Transport & Mean & 1700.66 & 1794.74 & 1534.21 & 0.71 & 2578.24 \\
\hline Equipment in RMB & Standard Deviation & 1822.07 & 1770.33 & 1922.34 & & 1697.82 \\
\hline & Number of Obs & 108 & 69 & 39 & & 21 \\
\hline
\end{tabular}

Notes: See Design/Data Section and Appendix F for discussion. 
Table A.5: Descriptive Statistics: Firm's Transaction Data

\begin{tabular}{|c|c|c|c|c|c|c|c|c|c|c|}
\hline & $\begin{array}{c}\text { Number of } \\
\text { Purchase } \\
\text { Transactions }\end{array}$ & $\begin{array}{l}\text { Number of } \\
\text { Buyers }\end{array}$ & $\begin{array}{l}\text { Number of Out- } \\
\text { Shipments }\end{array}$ & $\begin{array}{c}\text { Number of } \\
\text { Terminals }\end{array}$ & $\begin{array}{l}\text { Number of } \\
\text { Counties }\end{array}$ & $\begin{array}{l}\text { Number of } \\
\text { Provinces }\end{array}$ & $\begin{array}{l}\text { Number of } \\
\text { Days }\end{array}$ & $\begin{array}{l}\text { Number of } \\
\text { Months }\end{array}$ & $\begin{array}{c}\text { Sum of } \\
\text { Payments } \\
(\mathrm{RMB}) \\
\end{array}$ & $\begin{array}{c}\text { Sum of Out- } \\
\text { Shipments } \\
\text { (Weight in kg) }\end{array}$ \\
\hline Full Sample & $27,270,532$ & $3,785,019$ & 500,743 & 11,941 & 175 & 5 & 547 & 18 & $4,480,424,896$ & $1,169,673$ \\
\hline 3 Provinces & $20,647,373$ & $2,832,872$ & 442,319 & 8,561 & 116 & 3 & 547 & 18 & $3,409,227,245$ & $1,019,373$ \\
\hline 8 Counties & $1,835,897$ & 216,529 & 44,148 & 706 & 8 & 3 & 503 & 17 & $330,930,097$ & 95,908 \\
\hline RCT Villages & 130,769 & 15,099 & 3,158 & 40 & 8 & 3 & 482 & 16 & $17,618,900$ & 7,817 \\
\hline
\end{tabular}

Notes: The table provides information from the purchase and the sales transaction databases. The purchase database covers all village transactions in 5 provinces over the period November 2015 until April 2017. The sales transaction database covers all out-shipments from the same locations over the period January 2016 to April 2017. See Section Design/Data for discussion. 
Table A.6: Average Effects: Consumption

\begin{tabular}{|c|c|c|c|c|c|c|c|c|c|}
\hline Dependent Variables & & Intent to Treat & $\begin{array}{l}\text { Treatment on } \\
\text { Treated }\end{array}$ & $\begin{array}{l}\text { Log Distance } \\
\text { (IV using Treat) }\end{array}$ & Dependent Variables & & Intent to Treat & $\begin{array}{l}\text { Treatment on } \\
\text { Treated }\end{array}$ & $\begin{array}{l}\text { Log Distance } \\
\text { (IV using Treat) }\end{array}$ \\
\hline $\begin{array}{l}\text { Monthly Total Retail } \\
\text { Expenditure Per Capita }\end{array}$ & $\begin{array}{l}\text { Treat or Log Dist } \\
\text { R-Squared } \\
\text { First Stage F-Stat } \\
\text { Number of Obs }\end{array}$ & $\begin{array}{c}-22.09 \\
(31.99) \\
0.038 \\
\\
3,436\end{array}$ & $\begin{array}{l}-41.20 \\
(60.22) \\
\\
44.01 \\
3,436\end{array}$ & $\begin{array}{c}10.79 \\
(15.67) \\
\\
48.31 \\
3,436\end{array}$ & $\begin{array}{l}\text { Share of E-Comm Option } \\
\text { in Monthly Tobacco and } \\
\text { Alcohol (2) }\end{array}$ & $\begin{array}{l}\text { Treat or Log Dist } \\
\text { R-Squared } \\
\text { First Stage F-Stat } \\
\text { Number of Obs }\end{array}$ & $\begin{array}{c}0.000608 \\
(0.000515) \\
0.001\end{array}$ & $\begin{array}{c}0.00123 \\
(0.00109)\end{array}$ & $\begin{array}{l}-0.000330 \\
(0.000287)\end{array}$ \\
\hline $\begin{array}{l}\text { Household Has Ever Bought } \\
\text { Something through E-Comm } \\
\text { Option (Yes=1) }\end{array}$ & $\begin{array}{l}\text { Treat or Log Dist } \\
\text { R-Squared } \\
\text { First Stage F-Stat } \\
\text { Number of Obs }\end{array}$ & $\begin{array}{c}0.0484^{* * *} \\
(0.0167) \\
0.008\end{array}$ & $\begin{array}{c}0.0894^{* * *} \\
(0.0268)\end{array}$ & $\begin{array}{l}-0.0234 * * * \\
(0.00697)\end{array}$ & $\begin{array}{l}\text { Share of E-Comm Option } \\
\text { in Monthly Medicine and } \\
\text { Health Products (3) }\end{array}$ & $\begin{array}{l}\text { Treat or Log Dist } \\
\text { R-Squared } \\
\text { First Stage F-Stat } \\
\text { Number of Obs }\end{array}$ & $\begin{array}{c}0.000693 \\
(0.000689) \\
0.000\end{array}$ & $\begin{array}{c}0.00126 \\
(0.00124)\end{array}$ & $\begin{array}{l}-0.000329 \\
(0.000324)\end{array}$ \\
\hline $\begin{array}{l}\text { Household Has Bought } \\
\text { Something in Past Month } \\
\text { (Yes=1) }\end{array}$ & $\begin{array}{l}\text { Treat or Log Dist } \\
\text { R-Squared } \\
\text { First Stage F-Stat } \\
\text { Number of Obs }\end{array}$ & $\begin{array}{c}0.0263 * * * \\
(0.00981) \\
0.009 \\
\\
3,482 \\
\end{array}$ & $\begin{array}{c}0.0490^{* * *} \\
(0.0171)\end{array}$ & $\begin{array}{c}-0.0128^{* * *} \\
(0.00445) \\
\\
47.95 \\
3,482 \\
\end{array}$ & $\begin{array}{l}\text { Share of E-Comm Option } \\
\text { in Monthly Clothing and } \\
\text { Accessories (4) }\end{array}$ & $\begin{array}{l}\text { Treat or Log Dist } \\
\text { R-Squared } \\
\text { First Stage F-Stat } \\
\text { Number of Obs }\end{array}$ & $\begin{array}{c}0.0466^{* * *} \\
(0.0140) \\
0.019 \\
\\
1,268 \\
\end{array}$ & $\begin{array}{l}0.0736^{* * *} \\
(0.0217)\end{array}$ & $\begin{array}{c}-0.0201 * * * \\
(0.00594) \\
\\
65.25 \\
1,268 \\
\end{array}$ \\
\hline $\begin{array}{l}\text { Share of E-Comm Option in } \\
\text { Total Monthly Retail } \\
\text { Expenditure }\end{array}$ & $\begin{array}{l}\text { Treat or Log Dist } \\
\text { R-Squared } \\
\text { First Stage F-Stat } \\
\text { Number of Obs }\end{array}$ & $\begin{array}{c}0.00668^{* * *} \\
(0.00239) \\
0.006\end{array}$ & $\begin{array}{l}0.0124 * * * \\
(0.00435)\end{array}$ & $\begin{array}{l}-0.00326^{* * *} \\
(0.00114)\end{array}$ & $\begin{array}{l}\text { Share of E-Comm Option } \\
\text { in Monthly Other } \\
\text { Household Products (5) }\end{array}$ & $\begin{array}{l}\text { Treat or Log Dist } \\
\text { R-Squared } \\
\text { First Stage F-Stat } \\
\text { Number of Obs }\end{array}$ & $\begin{array}{c}0.00437 \\
(0.00396) \\
0.001 \\
\\
2,336\end{array}$ & $\begin{array}{c}0.00816 \\
(0.00715)\end{array}$ & $\begin{array}{c}-0.00217 \\
(0.00190) \\
\\
47.76 \\
2,336\end{array}$ \\
\hline $\begin{array}{l}\text { Share of E-Comm Option in } \\
\text { Monthly Business Inputs }\end{array}$ & $\begin{array}{l}\text { Treat or Log Dist } \\
\text { R-Squared } \\
\text { First Stage F-Stat } \\
\text { Number of Obs }\end{array}$ & $\begin{array}{c}-0.00707 \\
(0.00779) \\
0.003 \\
\\
1,191 \\
\end{array}$ & $\begin{array}{l}-0.0155 \\
(0.0195)\end{array}$ & $\begin{array}{c}0.00403 \\
(0.00507)\end{array}$ & $\begin{array}{l}\text { Share of E-Comm Option } \\
\text { in Monthly Heating, Fuel } \\
\text { and Gas (6) }\end{array}$ & $\begin{array}{l}\text { Treat or Log Dist } \\
\text { R-Squared } \\
\text { First Stage F-Stat } \\
\text { Number of Obs }\end{array}$ & $\begin{array}{c}0 \\
(0) \\
\cdot \\
1,463 \\
\end{array}$ & 1,463 & $\begin{array}{c}0 \\
(0) \\
\\
. \\
1,463 \\
\end{array}$ \\
\hline $\begin{array}{l}\text { Share of E-Comm Option in } \\
\text { Monthly Non-Durables }\end{array}$ & $\begin{array}{l}\text { Treat or Log Dist } \\
\text { R-Squared } \\
\text { First Stage F-Stat } \\
\text { Number of Obs }\end{array}$ & $\begin{array}{c}0.00538^{* * *} \\
(0.00196) \\
0.003 \\
\\
3,433\end{array}$ & $\begin{array}{c}0.0100^{* * *} \\
(0.00356) \\
\\
44.11 \\
3,433\end{array}$ & $\begin{array}{c}-0.00262^{* * *} \\
(0.000933) \\
48 \\
3,433\end{array}$ & $\begin{array}{l}\text { Share of E-Comm Option } \\
\text { in Monthly Furniture and } \\
\text { Appliances (7) }\end{array}$ & $\begin{array}{l}\text { Treat or Log Dist } \\
\text { R-Squared } \\
\text { First Stage F-Stat } \\
\text { Number of Obs }\end{array}$ & $\begin{array}{c}0.0546^{* *} \\
(0.0217) \\
0.019 \\
\\
380\end{array}$ & $\begin{array}{l}0.0908^{* *} \\
(0.0368)\end{array}$ & $\begin{array}{c}-0.0253^{* *} \\
(0.0101) \\
\\
42.04 \\
380\end{array}$ \\
\hline $\begin{array}{l}\text { Share of E-Comm Option in } \\
\text { Monthly Durables }\end{array}$ & $\begin{array}{l}\text { Treat or Log Dist } \\
\text { R-Squared } \\
\text { First Stage F-Stat } \\
\text { Number of Obs }\end{array}$ & $\begin{array}{c}0.0408^{* *} \\
(0.0160) \\
0.012 \\
\\
768 \\
\end{array}$ & $\begin{array}{c}0.0686^{* * *} \\
(0.0263) \\
\\
52.43 \\
768 \\
\end{array}$ & $\begin{array}{c}-0.0191 * * * \\
(0.00727) \\
\\
44.14 \\
768 \\
\end{array}$ & $\begin{array}{l}\text { Share of E-Comm Option } \\
\text { in Monthly Electronics ( } 8 \text { ) }\end{array}$ & $\begin{array}{l}\text { Treat or Log Dist } \\
\text { R-Squared } \\
\text { First Stage F-Stat } \\
\text { Number of Obs }\end{array}$ & $\begin{array}{c}0.0698^{* *} \\
(0.0347) \\
0.023 \\
\\
231 \\
\end{array}$ & $\begin{array}{c}0.111^{* *} \\
(0.0527) \\
\\
42.35 \\
231 \\
\end{array}$ & $\begin{array}{c}-0.0339^{* *} \\
(0.0159) \\
\\
26.54 \\
231 \\
\end{array}$ \\
\hline $\begin{array}{l}\text { Share of E-Comm Option in } \\
\text { Monthly Food and } \\
\text { Beverages (1) }\end{array}$ & $\begin{array}{l}\text { Treat or Log Dist } \\
\text { R-Squared } \\
\text { First Stage F-Stat } \\
\text { Number of Obs }\end{array}$ & $\begin{array}{c}0.00121 \\
(0.000823) \\
0.001\end{array}$ & $\begin{array}{c}0.00223 \\
(0.00152)\end{array}$ & $\begin{array}{l}-0.000582 \\
(0.000398)\end{array}$ & $\begin{array}{l}\text { Share of E-Comm Option } \\
\text { in Monthly Transport } \\
\text { Equipment (9) }\end{array}$ & $\begin{array}{l}\text { Treat or Log Dist } \\
\text { R-Squared } \\
\text { First Stage F-Stat } \\
\text { Number of Obs }\end{array}$ & $\begin{array}{c}0.0357^{*} \\
(0.0203) \\
0.014\end{array}$ & $\begin{array}{l}0.0565^{*} \\
(0.0319)\end{array}$ & $\begin{array}{c}-0.0152^{*} \\
(0.00878) \\
\\
42.37 \\
139\end{array}$ \\
\hline
\end{tabular}

Notes: Table reports point estimates from specification (1). The first column reports ITT and the second column TOT. The third column replaces the binary TOT with log residential distances to the nearest e-commerce terminal (using village-level ITT as instrument as for second column). Standard errors are clustered at the level of villages. ${ }^{*} 10 \%$, ${ }^{* *} 5 \%$, ${ }^{* * *} 1 \%$ significance levels. 
Table A.7: Average Effects: Incomes

\begin{tabular}{|c|c|c|c|c|c|c|c|c|c|}
\hline Dependent Variables & & Intent to Treat & $\begin{array}{l}\text { Treatment on } \\
\text { Treated }\end{array}$ & $\begin{array}{c}\text { Log Distance } \\
\text { (IV using Treat) }\end{array}$ & Dependent Variables & & Intent to Treat & $\begin{array}{c}\text { Treatment on } \\
\text { Treated }\end{array}$ & $\begin{array}{c}\text { Log Distance } \\
\text { (IV using Treat) }\end{array}$ \\
\hline \multirow{4}{*}{$\begin{array}{l}\text { Monthly Income Per } \\
\text { Capita in RMB }\end{array}$} & & $\begin{array}{l}-7.864 \\
(70.78)\end{array}$ & $\begin{array}{l}-14.53 \\
(129.9)\end{array}$ & $\begin{array}{c}3.974 \\
(35.61)\end{array}$ & \multirow{4}{*}{$\begin{array}{l}\text { Member of Household } \\
\text { Has Ever Sold through } \\
\text { E-Commerce (Yes=1) }\end{array}$} & & $\begin{array}{l}-0.00700 \\
(0.00562)\end{array}$ & $\begin{array}{l}-0.0129 \\
(0.0104)\end{array}$ & $\begin{array}{c}0.00353 \\
(0.00282)\end{array}$ \\
\hline & R-Squared & 0.038 & & & & R-Squared & 0.347 & & \\
\hline & First Stage F-Stat & & 45.33 & 42.83 & & First Stage F-Stat & & 45.30 & 42.71 \\
\hline & Number of Obs & 3,437 & 3,437 & 3,437 & & Number of Obs & 3,504 & 3,504 & 3,504 \\
\hline \multirow{4}{*}{$\begin{array}{l}\text { Monthly Income Per } \\
\text { Capita Net of Costs in } \\
\text { RMB }\end{array}$} & Treat or Log Dist & $\begin{array}{l}-20.09 \\
(70.80)\end{array}$ & $\begin{array}{l}-37.20 \\
(129.9)\end{array}$ & $\begin{array}{c}10.19 \\
(35.51)\end{array}$ & \multirow{4}{*}{$\begin{array}{l}\text { Member of Household } \\
\text { Has Sold through } \\
\text { E-Commerce In Past } \\
\text { Month (Yes=1) }\end{array}$} & Treat or Log Dist & $\begin{array}{l}-0.00132 \\
(0.00237)\end{array}$ & $\begin{array}{l}-0.00244 \\
(0.00438)\end{array}$ & $\begin{array}{l}0.000667 \\
(0.00119)\end{array}$ \\
\hline & R-Squared & 0.037 & & & & R-Squared & 0.038 & & \\
\hline & First Stage F-Stat & & 44.78 & 42.54 & & First Stage F-Stat & & 44.30 & 42.34 \\
\hline & Number of Obs & 3,390 & 3,390 & 3,390 & & Number of Obs & 3,498 & 3,498 & 3,498 \\
\hline \multirow{4}{*}{$\begin{array}{l}\text { Monthly Income Per } \\
\text { Capita Net of Transfers in } \\
\text { RMB }\end{array}$} & Treat or Log Dist & $\begin{array}{l}-12.55 \\
(72.18)\end{array}$ & $\begin{array}{l}-23.21 \\
(132.4)\end{array}$ & $\begin{array}{l}6.360 \\
(36.25)\end{array}$ & \multirow{4}{*}{$\begin{array}{l}\text { E-Commerce Sales in } \\
\text { Past Month in RMB }\end{array}$} & Treat or Log Dist & $\begin{array}{l}-10.09 \\
(12.89)\end{array}$ & $\begin{array}{l}-18.75 \\
(23.94)\end{array}$ & $\begin{array}{c}5.109 \\
(6.504)\end{array}$ \\
\hline & R-Squared & 0.051 & & & & R-Squared & 0.012 & & \\
\hline & First Stage F-Stat & & 45.16 & 42.67 & & First Stage F-Stat & & 44.26 & 42.39 \\
\hline & Number of Obs & 3,445 & 3,445 & 3,445 & & Number of Obs & 3,498 & 3,498 & 3,498 \\
\hline \multirow{4}{*}{$\begin{array}{l}\text { Annual Income Per Capita } \\
\text { in RMB }\end{array}$} & Treat or Log Dist & $\begin{array}{l}-45.95 \\
(586.9)\end{array}$ & $\begin{array}{l}-85.08 \\
(1,080)\end{array}$ & $\begin{array}{c}23.33 \\
(296.3)\end{array}$ & \multirow{4}{*}{$\begin{array}{l}\text { Share of E-Commerce } \\
\text { Sales in Household } \\
\text { Monthly Income }\end{array}$} & Treat or Log Dist & $\begin{array}{l}-0.00120 \\
(0.00176)\end{array}$ & $\begin{array}{l}-0.00224 \\
(0.00330)\end{array}$ & $\begin{array}{c}0.000614 \\
(0.000901)\end{array}$ \\
\hline & R-Squared & 0.046 & & & & R-Squared & 0.032 & & \\
\hline & First Stage F-Stat & & 44.77 & 42.23 & & First Stage F-Stat & & 41.62 & 38.41 \\
\hline & Number of Obs & 3,388 & 3,388 & 3,388 & & Number of Obs & 2,830 & 2,830 & 2,830 \\
\hline \multirow{4}{*}{$\begin{array}{l}\text { Monthly Agricultural } \\
\text { Income Per Capita }\end{array}$} & Treat or Log Dist & $\begin{array}{l}-70.23 \\
(140.3)\end{array}$ & $\begin{array}{l}-130.3 \\
(257.7)\end{array}$ & $\begin{array}{l}35.61 \\
(70.34)\end{array}$ & \multirow{4}{*}{$\begin{array}{l}\text { Primary Earner Working } \\
\text { As Farmer (Yes=1) }\end{array}$} & Treat or Log Dist & $\begin{array}{l}-0.0229 \\
(0.0319)\end{array}$ & $\begin{array}{l}-0.0425 \\
(0.0597)\end{array}$ & $\begin{array}{c}0.0116 \\
(0.0164)\end{array}$ \\
\hline & R-Squared & 0.033 & & & & R-Squared & 0.140 & & \\
\hline & First Stage F-Stat & & 44.23 & 42.33 & & First Stage F-Stat & & 44.42 & 41.58 \\
\hline & Number of Obs & 3,448 & 3,448 & 3,448 & & Number of Obs & 3,327 & 3,327 & 3,327 \\
\hline \multirow{4}{*}{$\begin{array}{l}\text { Monthly Non- } \\
\text { Agricultural Income Per } \\
\text { Capita }\end{array}$} & Treat or Log Dist & $\begin{array}{l}-46.65 \\
(137.3)\end{array}$ & $\begin{array}{l}-86.06 \\
(249.6)\end{array}$ & $\begin{array}{c}23.55 \\
(68.28)\end{array}$ & \multirow{4}{*}{$\begin{array}{l}\text { Member of Household } \\
\text { Started a Business Over } \\
\text { Last } 6 \text { Months (Yes=1) }\end{array}$} & Treat or Log Dist & $\begin{array}{l}-0.00802 \\
(0.00631)\end{array}$ & $\begin{array}{l}-0.0149 \\
(0.0120)\end{array}$ & $\begin{array}{c}0.00407 \\
(0.00327)\end{array}$ \\
\hline & R-Squared & 0.157 & & & & R-Squared & 0.001 & & \\
\hline & First Stage F-Stat & & 45.74 & 43.51 & & First Stage F-Stat & & 44.37 & 42.34 \\
\hline & Number of Obs & 3,441 & 3,441 & 3,441 & & Number of Obs & 3,468 & 3,468 & 3,468 \\
\hline \multirow{4}{*}{$\begin{array}{l}\text { Weekly Hours Worked by } \\
\text { Primary Earner }\end{array}$} & Treat or Log Dist & $\begin{array}{c}1.008 \\
(3.383)\end{array}$ & $\begin{array}{c}1.879 \\
(6.285)\end{array}$ & $\begin{array}{l}-0.516 \\
(1.723)\end{array}$ & \multirow{4}{*}{$\begin{array}{l}\text { New Business Selling in } \\
\text { Part Online (Yes=1) }\end{array}$} & Treat or Log Dist & $\begin{array}{l}0.000212 \\
(0.00159)\end{array}$ & $\begin{array}{l}0.000394 \\
(0.00294)\end{array}$ & $\begin{array}{l}-0.000108 \\
(0.000803)\end{array}$ \\
\hline & R-Squared & 0.000 & & & & R-Squared & 0.000 & & \\
\hline & First Stage F-Stat & & 43.80 & 41.21 & & First Stage F-Stat & & 44.33 & 42.37 \\
\hline & Number of Obs & 3,310 & 3,310 & 3,310 & & Number of Obs & 3,468 & 3,468 & 3,468 \\
\hline \multirow{4}{*}{$\begin{array}{l}\text { Weekly Hours Worked by } \\
\text { Secondary Earner }\end{array}$} & Treat or Log Dist & $\begin{array}{l}-0.0606 \\
(3.886)\end{array}$ & $\begin{array}{l}-0.110 \\
(7.002)\end{array}$ & $\begin{array}{l}0.0317 \\
(2.020)\end{array}$ & & & & & \\
\hline & R-Squared & 0.000 & & & & & & & \\
\hline & First Stage F-Stat & & 45.39 & 40.21 & & & & & \\
\hline & Number of Obs & 1,866 & 1,866 & 1,866 & & & & & \\
\hline
\end{tabular}

Notes: Table reports point estimates from specification (1). The first column reports ITT and the second column TOT. The third column replaces the binary TOT with log residential distances to the nearest e-commerce terminal (using village-level ITT as instrument as for second column). Standard errors are clustered at the level of villages. ${ }^{*} 10 \%$, $* * \%$, $* * * 1 \%$ significance levels. 
Table A.8: Average Effects: Local Retail Prices

\begin{tabular}{|c|c|c|c|c|c|c|c|}
\hline Dependent Variables & & Intent to Treat & $\begin{array}{c}\text { Treatment on } \\
\text { Treated }\end{array}$ & Dependent Variables & & Intent to Treat & $\begin{array}{c}\text { Treatment on } \\
\text { Treated }\end{array}$ \\
\hline Log Prices (All) & $\begin{array}{l}\text { Treat } \\
\text { R-Squared } \\
\text { First Stage F-Stat } \\
\text { Number of Obs }\end{array}$ & $\begin{array}{c}0.0189 \\
(0.0142) \\
0.893 \\
\\
6,877 \\
\end{array}$ & $\begin{array}{c}0.0352 \\
(0.0263) \\
0.893 \\
41.66 \\
6,877 \\
\end{array}$ & $\begin{array}{l}\text { Log Prices of Food } \\
\text { and Beverages (1) }\end{array}$ & $\begin{array}{l}\text { Treat } \\
\text { R-Squared } \\
\text { First Stage F-Stat } \\
\text { Number of Obs }\end{array}$ & $\begin{array}{c}0.0368 * * \\
(0.0185) \\
0.870 \\
\\
3,686\end{array}$ & $\begin{array}{c}0.0706^{*} \\
(0.0375) \\
0.870 \\
39.37 \\
3,686\end{array}$ \\
\hline $\begin{array}{l}\text { Product Replacement } \\
\text { Dummy (Not Counting } \\
\text { Store Closures) } \\
(\text { Yes }=1)\end{array}$ & $\begin{array}{l}\text { Treat } \\
\text { R-Squared } \\
\text { First Stage F-Stat } \\
\text { Number of Obs }\end{array}$ & $\begin{array}{c}-0.00516 \\
(0.00947) \\
0.000 \\
\\
8,956\end{array}$ & $\begin{array}{c}-0.00983 \\
(0.0181) \\
-0.002 \\
39.82 \\
8,956\end{array}$ & $\begin{array}{l}\text { Log Prices of Tobacco } \\
\text { and Alcohol (2) }\end{array}$ & $\begin{array}{l}\text { Treat } \\
\text { R-Squared } \\
\text { First Stage F-Stat } \\
\text { Number of Obs }\end{array}$ & $\begin{array}{c}0.0212 \\
(0.0340) \\
0.809\end{array}$ & $\begin{array}{c}0.0421 \\
(0.0662) \\
0.810 \\
32.39 \\
1,071\end{array}$ \\
\hline $\begin{array}{l}\text { Store Closure (at } \\
\text { Product Level) }(\text { Yes }=1)\end{array}$ & $\begin{array}{l}\text { Treat } \\
\text { R-Squared } \\
\text { First Stage F-Stat } \\
\text { Number of Obs }\end{array}$ & $\begin{array}{c}0.00124 \\
(0.0294) \\
0.000 \\
\\
8,956 \\
\end{array}$ & $\begin{array}{c}0.00236 \\
(0.0556) \\
0.000 \\
39.82 \\
8,956 \\
\end{array}$ & $\begin{array}{l}\text { Log Prices of } \\
\text { Medicine and Health } \\
\text { Products (3) }\end{array}$ & $\begin{array}{l}\text { Treat } \\
\text { R-Squared } \\
\text { First Stage F-Stat } \\
\text { Number of Obs }\end{array}$ & $\begin{array}{c}-0.0474 \\
(0.0741) \\
0.794 \\
\\
266 \\
\end{array}$ & $\begin{array}{c}-0.0756 \\
(0.122) \\
0.795 \\
19.18 \\
266 \\
\end{array}$ \\
\hline $\begin{array}{l}\text { Number of New } \\
\text { Products Per Store }\end{array}$ & $\begin{array}{l}\text { Treat } \\
\text { R-Squared } \\
\text { First Stage F-Stat } \\
\text { Number of Obs }\end{array}$ & $\begin{array}{c}2.194 * * \\
(1.073) \\
0.277 \\
\\
312 \\
\end{array}$ & $\begin{array}{c}4.020^{*} \\
(2.278) \\
0.212 \\
19.69 \\
312 \\
\end{array}$ & $\begin{array}{l}\text { Log Prices of } \\
\text { Clothing and } \\
\text { Accessories (4) }\end{array}$ & $\begin{array}{l}\text { Treat } \\
\text { R-Squared } \\
\text { First Stage F-Stat } \\
\text { Number of Obs }\end{array}$ & $\begin{array}{c}0.0809 \\
(0.111) \\
0.845 \\
\\
152 \\
\end{array}$ & $\begin{array}{c}0.115 \\
(0.158) \\
0.842 \\
42.80 \\
152 \\
\end{array}$ \\
\hline $\begin{array}{l}\text { Store Owner Sources } \\
\text { Products Online } \\
(\mathrm{Yes}=1)\end{array}$ & $\begin{array}{l}\text { Treat } \\
\text { R-Squared } \\
\text { First Stage F-Stat } \\
\text { Number of Obs }\end{array}$ & $\begin{array}{c}-0.00145 \\
(0.0258) \\
0.000 \\
\\
341 \\
\end{array}$ & $\begin{array}{c}-0.00261 \\
(0.0461) \\
-0.001 \\
23.76 \\
341 \\
\end{array}$ & $\begin{array}{l}\text { Log Prices of Other } \\
\text { Household Products } \\
\text { (5) }\end{array}$ & $\begin{array}{l}\text { Treat } \\
\text { R-Squared } \\
\text { First Stage F-Stat } \\
\text { Number of Obs }\end{array}$ & $\begin{array}{c}-0.0328 \\
(0.0382) \\
0.756 \\
\\
1,268 \\
\end{array}$ & $\begin{array}{c}-0.0619 \\
(0.0744) \\
0.755 \\
28.85 \\
1,268 \\
\end{array}$ \\
\hline $\begin{array}{l}\text { Log Prices of Business } \\
\text { Inputs }\end{array}$ & $\begin{array}{l}\text { Treat } \\
\text { R-Squared } \\
\text { First Stage F-Stat } \\
\text { Number of Obs }\end{array}$ & $\begin{array}{c}0.00229 \\
(0.129) \\
0.811 \\
\\
237 \\
\end{array}$ & $\begin{array}{c}0.00337 \\
(0.186) \\
0.811 \\
24.86 \\
237 \\
\end{array}$ & $\begin{array}{l}\text { Log Prices of Heating, } \\
\text { Fuel and Gas (6) }\end{array}$ & $\begin{array}{l}\text { Treat } \\
\text { R-Squared } \\
\text { First Stage F-Stat } \\
\text { Number of Obs }\end{array}$ & $\begin{array}{c}-0.0115 \\
(0.0955) \\
0.007 \\
\\
12 \\
\end{array}$ & $\begin{array}{c}-0.0440 \\
(0.332) \\
-0.095 \\
0.795 \\
12 \\
\end{array}$ \\
\hline $\begin{array}{l}\text { Log Prices of Non- } \\
\text { Durables }\end{array}$ & $\begin{array}{l}\text { Treat } \\
\text { R-Squared } \\
\text { First Stage F-Stat } \\
\text { Number of Obs }\end{array}$ & $\begin{array}{c}0.0211 \\
(0.0146) \\
0.860 \\
\\
6,455 \\
\end{array}$ & $\begin{array}{c}0.0398 \\
(0.0276) \\
0.860 \\
40.36 \\
6,455 \\
\end{array}$ & $\begin{array}{l}\text { Log Prices of } \\
\text { Furniture and } \\
\text { Appliances (7) }\end{array}$ & $\begin{array}{l}\text { Treat } \\
\text { R-Squared } \\
\text { First Stage F-Stat } \\
\text { Number of Obs }\end{array}$ & $\begin{array}{c}-0.0347 \\
(0.0881) \\
0.952 \\
\\
109 \\
\end{array}$ & $\begin{array}{c}-0.0617 \\
(0.156) \\
0.953 \\
6.757 \\
109 \\
\end{array}$ \\
\hline Log Prices of Durables & $\begin{array}{l}\text { Treat } \\
\text { R-Squared } \\
\text { First Stage F-Stat } \\
\text { Number of Obs }\end{array}$ & $\begin{array}{c}-0.0320 \\
(0.0711) \\
0.951 \\
\\
185\end{array}$ & $\begin{array}{c}-0.0522 \\
(0.115) \\
0.952 \\
9.753 \\
185\end{array}$ & $\begin{array}{l}\text { Log Prices of } \\
\text { Electronics }(8)\end{array}$ & $\begin{array}{l}\text { Treat } \\
\text { R-Squared } \\
\text { First Stage F-Stat } \\
\text { Number of Obs }\end{array}$ & $\begin{array}{c}-0.0892 \\
(0.305) \\
0.884 \\
\\
23\end{array}$ & $\begin{array}{c}-0.163 \\
(0.570) \\
0.890 \\
3.180 \\
23\end{array}$ \\
\hline & & & & $\begin{array}{l}\text { Log Prices of } \\
\text { Transport Equipment } \\
\text { (9) }\end{array}$ & $\begin{array}{l}\text { Treat } \\
\text { R-Squared } \\
\text { First Stage F-Stat } \\
\text { Number of Obs }\end{array}$ & $\begin{array}{c}0.0297 \\
(0.0840) \\
0.946 \\
53\end{array}$ & $\begin{array}{c}0.0398 \\
(0.110) \\
0.946 \\
22.67 \\
53\end{array}$ \\
\hline
\end{tabular}

Notes: Table reports point estimates from specification (1). The first column reports ITT and the second column TOT (using village-level ITT as instrument). Standard errors are clustered at the level of villages. ${ }^{*} 10 \%,{ }^{* *} 5 \%,{ }^{* * *} 1 \%$ significance levels. 
Table A.9: Role of Logistical and Transactional Barriers

\begin{tabular}{|c|c|c|c|c|c|c|c|c|c|c|c|c|c|}
\hline \multicolumn{5}{|c|}{ Effects on Consumption } & \multicolumn{5}{|c|}{ Effects on Incomes } & \multicolumn{4}{|c|}{ Effects on Retail Prices } \\
\hline Dept Variables & & Intent to Treat & $\begin{array}{c}\text { Treatment on the } \\
\text { Treated }\end{array}$ & $\begin{array}{c}\text { Log Distance } \\
\text { (IV Using Treat) }\end{array}$ & Dept Variables & & Intent to Treat & $\begin{array}{c}\text { Treatment on the } \\
\text { Treated }\end{array}$ & $\begin{array}{c}\text { Log Distance } \\
\text { (IV Using Treat) }\end{array}$ & Dept Variables & & Intent to Treat & $\begin{array}{l}\text { Treatment on the } \\
\text { Treated }\end{array}$ \\
\hline $\begin{array}{l}\text { Monthly Total Retail } \\
\text { Expenditure Per } \\
\text { Capita }\end{array}$ & $\begin{array}{l}\text { Treat or Log Dist } \\
\text { Treat or Log Dist * } \\
\text { Delivery } \\
\text { First Stage F-Stat } \\
\text { Number of Obs }\end{array}$ & $\begin{array}{c}-26.91 \\
(36.29) \\
31.64 \\
(69.36)\end{array}$ & $\begin{array}{c}-49.34 \\
(68.00) \\
58.94 \\
(140.5) \\
2.388 \\
3,436\end{array}$ & $\begin{array}{c}13.67 \\
(18.71) \\
-15.50 \\
(30.43) \\
19.39 \\
3,436\end{array}$ & $\begin{array}{l}\text { Monthly Income } \\
\text { Per Capita in RMB }\end{array}$ & $\begin{array}{l}\text { Treat or Log Dist } \\
\text { Treat or Log Dist * } \\
\text { Delivery } \\
\text { First Stage F-Stat } \\
\text { Number of Obs }\end{array}$ & $\begin{array}{c}-15.00 \\
(77.55) \\
50.17 \\
(171.1)\end{array}$ & $\begin{array}{c}-27.15 \\
(140.1) \\
96.91 \\
(339.0) \\
2.694 \\
3,437\end{array}$ & $\begin{array}{c}7.579 \\
(39.08) \\
-25.08 \\
(86.90) \\
2.737 \\
3,437\end{array}$ & Log Prices (All) & $\begin{array}{l}\text { Treat * Delivery } \\
\text { First Stage F-Stat } \\
\text { Number of Obs }\end{array}$ & $\begin{array}{c}0.0114 \\
(0.0144) \\
0.0417 \\
(0.0377)\end{array}$ & $\begin{array}{c}0.0215 \\
(0.0273) \\
0.0739 \\
(0.0572) \\
17.26 \\
6,877\end{array}$ \\
\hline $\begin{array}{l}\text { Household Has Ever } \\
\text { Bought Something } \\
\text { through E-Comm } \\
\text { Option (Yes=1) }\end{array}$ & $\begin{array}{l}\text { Treat or Log Dist } \\
\text { Treat or Log Dist * } \\
\text { Delivery } \\
\text { First Stage F-Stat } \\
\text { Number of Obs } \\
\end{array}$ & $\begin{array}{c}0.0578^{* * * *} \\
(0.0188) \\
-0.0606^{* *} \\
(0.0253)\end{array}$ & $\begin{array}{c}0.106 * * * \\
(0.0283) \\
-0.111 * * \\
(0.0443) \\
2.682 \\
3,518 \\
\end{array}$ & $\begin{array}{c}-0.0293 * * * \\
(0.00775) \\
0.0304 * * * \\
(0.0102) \\
19.63 \\
3,518 \\
\end{array}$ & $\begin{array}{l}\text { Monthly Income } \\
\text { Per Capita Net of } \\
\text { Costs in RMB }\end{array}$ & $\begin{array}{l}\text { Treat or Log Dist } \\
\text { Treat or Log Dist * } \\
\text { Delivery } \\
\text { First Stage F-Stat } \\
\text { Number of Obs }\end{array}$ & $\begin{array}{c}-20.24 \\
(77.47) \\
6.011 \\
(167.6)\end{array}$ & $\begin{array}{c}-37.09 \\
(140.5) \\
9.303 \\
(317.4) \\
2.810 \\
3,390\end{array}$ & $\begin{array}{c}10.33 \\
(39.07) \\
-3.362 \\
(81.28) \\
2.852 \\
3,390\end{array}$ & $\begin{array}{l}\text { Product } \\
\text { Replacement } \\
\text { Dummy (Not } \\
\text { Counting Store } \\
\text { Closures) (Yes=1) }\end{array}$ & $\begin{array}{l}\text { Treat } \\
\text { Treat * Delivery } \\
\text { First Stage F-Stat } \\
\text { Number of Obs }\end{array}$ & $\begin{array}{l}-0.00680 \\
(0.0108) \\
0.00907 \\
(0.0213)\end{array}$ & $\begin{array}{c}-0.0129 \\
(0.0206) \\
0.0173 \\
(0.0415) \\
2.648 \\
8,956\end{array}$ \\
\hline $\begin{array}{l}\text { Household Has } \\
\text { Bought Something in } \\
\text { Last Month (Yes=1) }\end{array}$ & $\begin{array}{l}\text { Treat or Log Dist } \\
\text { Treat or Log Dist * } \\
\text { Delivery } \\
\text { First Stage F-Stat } \\
\text { Number of Obs }\end{array}$ & $\begin{array}{c}0.0329 * * * \\
(0.0111) \\
-0.0422^{* * *} \\
(0.0155) \\
3,482\end{array}$ & $\begin{array}{c}0.0604^{* * *} \\
(0.0189) \\
-0.0790^{* *} \\
(0.0329) \\
2.513 \\
3,482\end{array}$ & $\begin{array}{c}-0.0168 * * * \\
(0.00522) \\
0.0204 * * * \\
(0.00729) \\
19.10 \\
3,482\end{array}$ & $\begin{array}{l}\text { Monthly Income } \\
\text { Per Capita Net of } \\
\text { Transfers in RMB }\end{array}$ & $\begin{array}{l}\text { Treat or Log Dist } \\
\text { Treat or Log Dist * } \\
\text { Delivery } \\
\text { First Stage F-Stat } \\
\text { Number of Obs }\end{array}$ & $\begin{array}{c}-13.87 \\
(77.86) \\
12.70 \\
(188.3)\end{array}$ & $\begin{array}{c}-25.27 \\
(140.7) \\
23.04 \\
(367.2) \\
2.635 \\
3,445\end{array}$ & $\begin{array}{c}7.041 \\
(39.18) \\
-6.473 \\
(93.22) \\
2.696 \\
3,445\end{array}$ & $\begin{array}{l}\text { Store Closure (at } \\
\text { Product Level) } \\
\text { (Yes=1) }\end{array}$ & $\begin{array}{l}\text { Treat * Delivery } \\
\text { First Stage F-Stat } \\
\text { Number of Obs }\end{array}$ & $\begin{array}{l}0.00111 \\
(0.0355) \\
0.000779 \\
(0.0423)\end{array}$ & $\begin{array}{c}0.00209 \\
(0.0668) \\
0.00162 \\
(0.0805) \\
2.648 \\
8,956\end{array}$ \\
\hline $\begin{array}{l}\text { Share of E-Comm } \\
\text { Option in Total } \\
\text { Monthly Retail } \\
\text { Expenditure }\end{array}$ & $\begin{array}{l}\text { Treat or Log Dist } \\
\text { Treat or Log Dist * } \\
\text { Delivery } \\
\text { First Stage F-Stat } \\
\text { Number of Obs }\end{array}$ & $\begin{array}{c}0.00799^{* * *} \\
(0.00275) \\
-0.00835^{* * *} \\
(0.00295)\end{array}$ & $\begin{array}{c}0.0147 * * * \\
(0.00489) \\
-0.0154 * * * \\
(0.00543) \\
2.413 \\
3,434\end{array}$ & $\begin{array}{c}-0.00407 * * * \\
(0.00136) \\
0.00422^{* * *} \\
(0.00144) \\
19.25 \\
3,434\end{array}$ & $\begin{array}{l}\text { Annual Income Per } \\
\text { Capita in RMB }\end{array}$ & $\begin{array}{l}\text { Treat or Log Dist } \\
\text { Treat or Log Dist * } \\
\text { Delivery } \\
\text { First Stage F-Stat } \\
\text { Number of Obs }\end{array}$ & $\begin{array}{c}70.33 \\
(645.0) \\
-734.1 \\
(1,484)\end{array}$ & $\begin{array}{c}124.2 \\
(1,168) \\
-1,462 \\
(2,755) \\
2.501 \\
3,388\end{array}$ & $\begin{array}{c}-34.68 \\
(325.6) \\
368.3 \\
(692.5) \\
2.603 \\
3,388\end{array}$ & $\begin{array}{l}\text { Number of New } \\
\text { Products Per Store }\end{array}$ & $\begin{array}{l}\text { Treat * Delivery } \\
\text { First Stage F-Stat } \\
\text { Number of Obs }\end{array}$ & $\begin{array}{c}1.403^{*} \\
(0.828) \\
3.403 \\
(3.876)\end{array}$ & $\begin{array}{c}2.352^{*} \\
(1.354) \\
7.993 \\
(12.77) \\
1.247 \\
312\end{array}$ \\
\hline $\begin{array}{l}\text { Share of E-Comm } \\
\text { Option in Total } \\
\text { Monthly Business } \\
\text { Inputs }\end{array}$ & $\begin{array}{l}\text { Treat or Log Dist } \\
\text { Treat or Log Dist * } \\
\text { Delivery } \\
\text { First Stage F-Stat } \\
\text { Number of Obs }\end{array}$ & $\begin{array}{c}-0.00830 \\
(0.00827) \\
0.0179 \\
(0.0113)\end{array}$ & $\begin{array}{c}-0.0190 \\
(0.0222) \\
0.0334 \\
(0.0250) \\
6.346 \\
1,191 \\
\end{array}$ & $\begin{array}{c}0.00501 \\
(0.00589) \\
-0.00818 \\
(0.00633) \\
7.094 \\
1,191 \\
\end{array}$ & $\begin{array}{l}\text { Member of } \\
\text { Household Has } \\
\text { Ever Sold through } \\
\text { E-Commerce } \\
\text { (Yes=1) }\end{array}$ & $\begin{array}{l}\text { First Stage F-Stat } \\
\text { Number of Obs }\end{array}$ & $\begin{array}{c}-0.00857 \\
(0.00608) \\
0.0102 \\
(0.0141)\end{array}$ & $\begin{array}{c}-0.0156 \\
(0.0111) \\
0.0188 \\
(0.0280) \\
2.561 \\
3,504\end{array}$ & $\begin{array}{c}0.00433 \\
(0.00309) \\
-0.00513 \\
(0.00715) \\
2.598 \\
3,504 \\
\end{array}$ & $\begin{array}{l}\text { Store Owner } \\
\text { Sources Products } \\
\text { Online (Yes=1) }\end{array}$ & $\begin{array}{l}\text { Treat * Delivery } \\
\text { First Stage F-Stat } \\
\text { Number of Obs }\end{array}$ & $\begin{array}{l}0.0250^{* *} \\
(0.0122) \\
-0.0911 \\
(0.0814)\end{array}$ & $\begin{array}{c}0.0416^{* * *} \\
(0.0201) \\
-0.185 \\
(0.166) \\
1.320 \\
341\end{array}$ \\
\hline $\begin{array}{l}\text { Share of E-Comm } \\
\text { Option in Total } \\
\text { Monthly Non- } \\
\text { Durables }\end{array}$ & $\begin{array}{l}\text { Treat or Log Dist } \\
\text { Treat or Log Dist * } \\
\text { Delivery } \\
\text { First Stage F-Stat } \\
\text { Number of Obs }\end{array}$ & $\begin{array}{c}0.00639^{* * *} \\
(0.00225) \\
-0.00648 * * \\
(0.00247)\end{array}$ & $\begin{array}{c}0.0117^{* * * *} \\
(0.00401) \\
-0.0119^{* * *} \\
(0.00453) \\
2.413 \\
3,433 \\
\end{array}$ & $\begin{array}{c}-0.00325^{* * *} \\
(0.00112) \\
0.00329^{* * *} \\
(0.00119) \\
19.26 \\
3,433 \\
\end{array}$ & $\begin{array}{l}\text { Share of } \\
\text { E-Commerce Sales } \\
\text { in Household } \\
\text { Monthly Income }\end{array}$ & $\begin{array}{l}\text { Treat or Log Dist * } \\
\text { First Stage F-Stat } \\
\text { Number of Obs }\end{array}$ & $\begin{array}{c}-0.00172 \\
(0.00210) \\
0.00282 \\
(0.00233)\end{array}$ & $\begin{array}{c}-0.00316 \\
(0.00387) \\
0.00540 \\
(0.00441) \\
2.402 \\
2,830 \\
\end{array}$ & $\begin{array}{c}0.000882 \\
(0.00108) \\
-0.00145 \\
(0.00121) \\
2.342 \\
2,830 \\
\end{array}$ & $\begin{array}{l}\text { Log Price of } \\
\text { Business Inputs }\end{array}$ & $\begin{array}{l}\text { Treat * Delivery } \\
\text { First Stage F-Stat } \\
\text { Number of Obs }\end{array}$ & $\begin{array}{c}-0.0858 \\
(0.134) \\
0.289 \\
(0.273)\end{array}$ & $\begin{array}{c}-0.108 \\
(0.182) \\
0.473 \\
(0.447) \\
1.972 \\
237\end{array}$ \\
\hline $\begin{array}{l}\text { Share of E-Comm } \\
\text { Option in Total } \\
\text { Monthly Durables }\end{array}$ & $\begin{array}{l}\text { Treat or Log Dist } \\
\text { Treat or Log Dist * } \\
\text { Delivery } \\
\text { First Stage F-Stat } \\
\text { Number of Obs }\end{array}$ & $\begin{array}{c}0.0497 * * * \\
(0.0177) \\
-0.0705 * * * \\
(0.0258) \\
768\end{array}$ & $\begin{array}{c}0.0825^{* * *} \\
(0.0286) \\
-0.120^{* * *} \\
(0.0443) \\
3.150 \\
768\end{array}$ & $\begin{array}{c}-0.0240 * * * \\
(0.00823) \\
0.0322 * * * \\
(0.0113) \\
18.33 \\
768\end{array}$ & $\begin{array}{l}\text { Primary Earner } \\
\text { Working as Peasant } \\
\text { (Yes=1) }\end{array}$ & $\begin{array}{l}\text { Treat or Log Dist * } \\
\text { First Stage F-Stat } \\
\text { Number of Obs }\end{array}$ & $\begin{array}{l}-0.0192 \\
(0.0341) \\
-0.0284 \\
(0.0813)\end{array}$ & $\begin{array}{c}-0.0352 \\
(0.0624) \\
-0.0609 \\
(0.185) \\
2.503 \\
3,327\end{array}$ & $\begin{array}{c}0.00979 \\
(0.0174) \\
0.0143 \\
(0.0464) \\
2.533 \\
3,327\end{array}$ & $\begin{array}{l}\text { Log Price of Non- } \\
\text { Durables }\end{array}$ & $\begin{array}{l}\text { Treat * Delivery } \\
\text { First Stage F-Stat } \\
\text { Number of Obs }\end{array}$ & $\begin{array}{c}0.0192 \\
(0.0157) \\
0.0137 \\
(0.0362)\end{array}$ & $\begin{array}{c}0.0366 \\
(0.0308) \\
0.0214 \\
(0.0585) \\
16.09 \\
6,455\end{array}$ \\
\hline & & & & & $\begin{array}{l}\text { Member of } \\
\text { Household Has } \\
\text { Started a Business } \\
\text { Over Last } 6 \text { Months } \\
\text { (Yes=1) }\end{array}$ & $\begin{array}{l}\text { Treat or Log Dist } \\
\text { Treat or Log Dist * } \\
\text { Delivery } \\
\text { First Stage F-Stat } \\
\text { Number of Obs }\end{array}$ & $\begin{array}{c}-0.0297 \\
(0.0183) \\
3,468\end{array}$ & $\begin{array}{c}-0.00601 \\
(0.0116) \\
\\
-0.0604 \\
(0.0536) \\
2.517 \\
3,468 \\
\end{array}$ & $\begin{array}{c}0.00167 \\
(0.00322) \\
\\
0.0149 \\
(0.0130) \\
2.566 \\
3,468 \\
\end{array}$ & $\begin{array}{l}\text { Log Prices of } \\
\text { Durables }\end{array}$ & $\begin{array}{l}\text { Treat } \\
\text { Treat * Delivery } \\
\text { First Stage F-Stat } \\
\text { Number of Obs }\end{array}$ & $\begin{array}{c}-0.118 \\
(0.0880)\end{array}$ & $\begin{array}{c}-0.144 \\
(0.104) \\
\\
0.288 \\
(0.366) \\
0.488 \\
185 \\
\end{array}$ \\
\hline
\end{tabular}

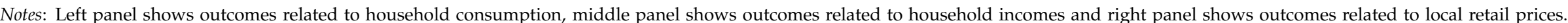

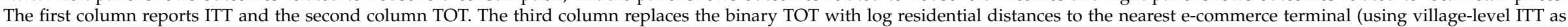
instrument as for second column). Standard errors are clustered at the level of villages. ${ }^{*} 10 \%, * * 5 \%,{ }^{* * *} 1 \%$ significance levels. 
Table A.10: Role of GE Spillovers

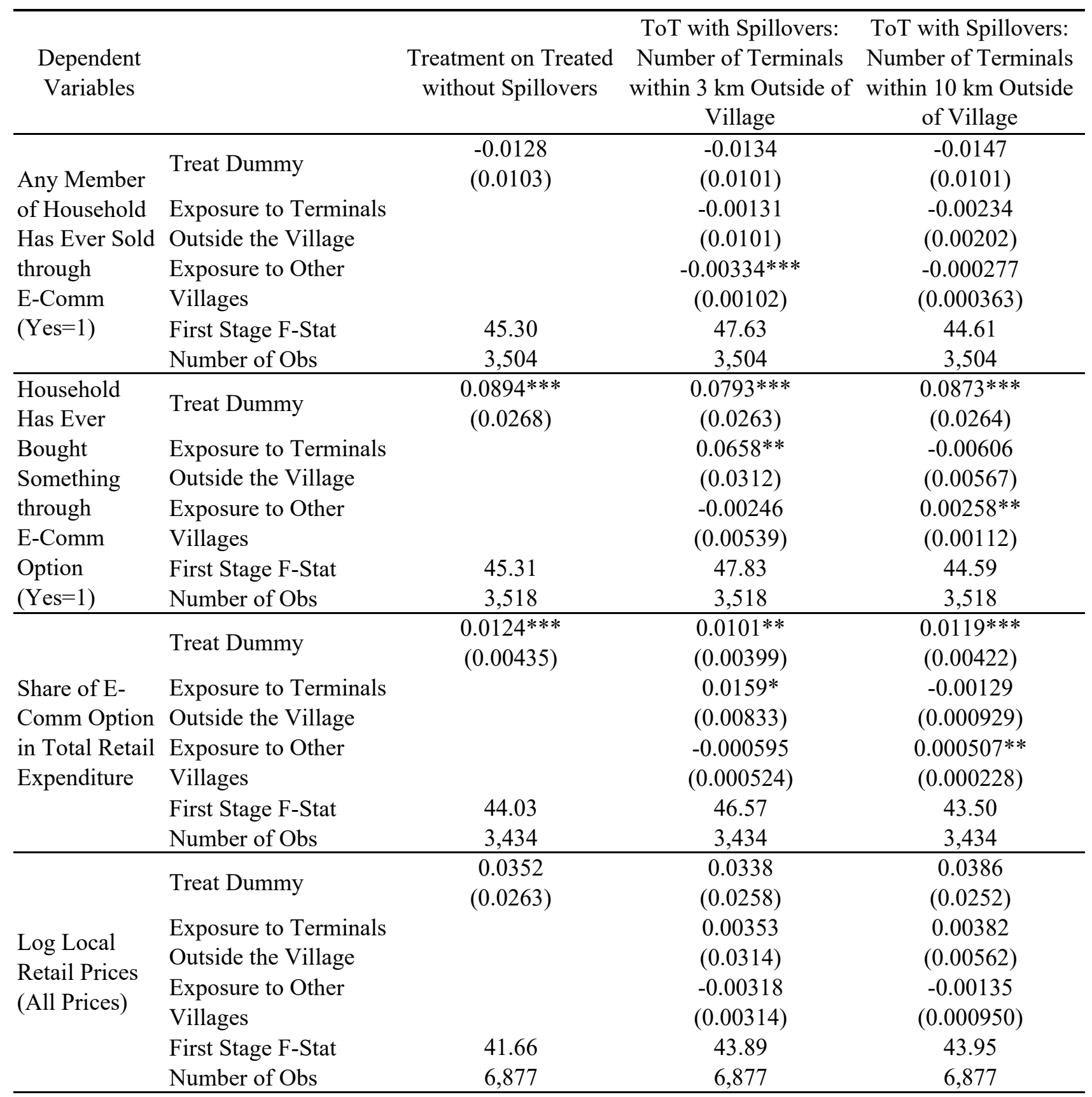

Notes: The first column reports the baseline TOT. The second column adds exposure to other intent-to-treat villages within a $3 \mathrm{~km}$ radius, controlling for the total number of eligible villages within this radius. The third column adds exposure to other intent-to-treat villages within a $10 \mathrm{~km}$ radius, controlling for the total number of eligible villages within this radius. See Appendix $\mathrm{C}$ for discussion. Standard errors are clustered at the level of villages. ${ }^{*} 10 \%,{ }^{* *} 5 \%,{ }^{* *} 1 \%$ significance levels. 
Table A.11: Fraction of Market Access to Other Rural Markets in County

\begin{tabular}{|c|c|c|c|c|c|c|c|c|c|c|c|c|}
\hline \multirow{3}{*}{ Measure of Market Size: } & \multicolumn{6}{|c|}{$\begin{array}{c}\text { Fraction of Market Access from Rural Markets in Same } \\
\text { County }\end{array}$} & \multicolumn{6}{|c|}{$\begin{array}{c}\text { Fraction of Market Access from Participating Rural } \\
\text { Markets in Same County }\end{array}$} \\
\hline & \multicolumn{3}{|c|}{ Access to Population } & \multicolumn{3}{|c|}{ Access to GDP } & \multicolumn{3}{|c|}{ Access to Population } & \multicolumn{3}{|c|}{ Access to GDP } \\
\hline & Median & Mean & Std Dev & Median & Mean & Std Dev & Median & Mean & Std Dev & Median & Mean & Std Dev \\
\hline \multicolumn{13}{|c|}{ Panel A: Distance Elasticity of -1} \\
\hline $\begin{array}{l}\text { Rural Townships in } 3 \text { RCT Provinces } \\
(2,291 \text { Townships })\end{array}$ & 0.012 & 0.016 & 0.014 & 0.0037 & 0.0059 & 0.0062 & 0.0020 & 0.0027 & 0.0023 & 0.0006 & 0.0010 & 0.0010 \\
\hline $\begin{array}{l}\text { Rural Townships in } 8 \text { RCT Counties } \\
\text { (58 Townships) }\end{array}$ & 0.011 & 0.012 & 0.006 & 0.0031 & 0.0041 & 0.0029 & 0.0018 & 0.0020 & 0.0010 & 0.0005 & 0.0007 & 0.0005 \\
\hline $\begin{array}{l}\text { Rural Townships in } 3 \text { RCT Provinces } \\
(2,291 \text { Townships })\end{array}$ & 0.036 & 0.049 & 0.055 & 0.012 & 0.02 & 0.028 & 0.0060 & 0.0082 & 0.0092 & 0.0020 & 0.0033 & 0.0047 \\
\hline $\begin{array}{l}\text { Rural Townships in } 8 \text { RCT Counties } \\
\text { (58 Townships) }\end{array}$ & 0.034 & 0.038 & 0.033 & 0.011 & 0.014 & 0.013 & 0.0057 & 0.0063 & 0.0055 & 0.0018 & 0.0023 & 0.0022 \\
\hline
\end{tabular}

Notes: Table reports the mean, median and standard deviation of the fraction of trade market access coming from other rural markets in the same county. See Appendix $\mathrm{C}$ for discussion. 
Table A.12: Are Sample Villages Representative?

(1) (2)

Dependent Variables:
Full Sample

(3)

Number of Transactions
(4)

(5)

3 Provinces
(6)

Sales (RMB) Number of Users Number of Transactions Sales (RMB)

\begin{tabular}{|c|c|c|c|c|c|c|}
\hline \multirow{3}{*}{ RCT_Sample Dummy } & \multicolumn{4}{|c|}{ Panel A: Purchase Database } & \multirow{3}{*}{$\begin{array}{c}12.65 \\
(25.32)\end{array}$} & \multirow{3}{*}{$\begin{array}{l}-3,747 \\
(4,066)\end{array}$} \\
\hline & -4.110 & 0.0605 & $-6,034$ & 0.149 & & \\
\hline & $(7.751)$ & $(25.33)$ & $(4,061)$ & $(7.734)$ & & \\
\hline Months Fixed Effects & $\checkmark$ & $\checkmark$ & $\checkmark$ & $\checkmark$ & $\checkmark$ & $\checkmark$ \\
\hline Control for Months Since Program Entry & $\checkmark$ & $\checkmark$ & $\checkmark$ & $\checkmark$ & $\checkmark$ & $\checkmark$ \\
\hline Observations & 125,204 & 125,204 & 125,204 & 100,098 & 100,098 & 100,098 \\
\hline R-squared & 0.037 & 0.047 & 0.029 & 0.031 & 0.046 & 0.03 \\
\hline Number of Village Clusters & 11,731 & 11,731 & 11,731 & 8,471 & 8,471 & 8,471 \\
\hline
\end{tabular}

(7)

Full Sample

(8)

(9)

3 Provinces

$\vec{\omega}$ Dependent Variables: Number of Transactions

Weight $(\mathrm{kg})$

Number of Transactions

(10)

RCT_Sample Dummy

Months Fixed Effects

Control for Months Since Program Entry

Observations

R-squared

Number of Village Clusters
$1.712 * *$

(0.753)

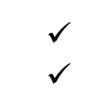

120,483

0.06

Panel B: Out-Shipment Database

$\begin{array}{ccc}5.154 & 1.364^{*} & 4.68 \\ (4.332) & (0.752) & (4.333)\end{array}$

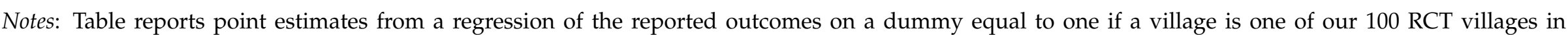

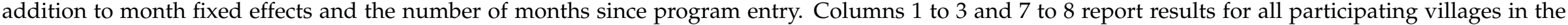

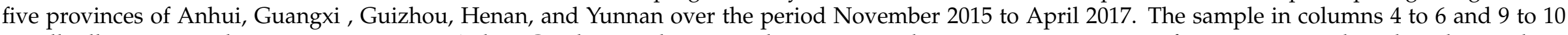

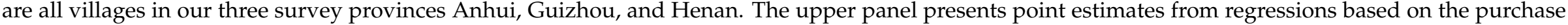

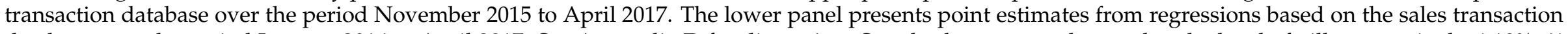

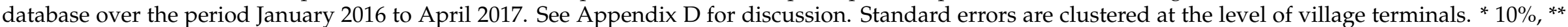
$5 \%, * * * 1 \%$ significance levels. 
Table A.13: Role of Seasonality

Full Sample

(3)

(4)

(5)

(6)

$\underline{\text { Dependent Variables: }}$

Number of Users

Number of Transactions

Sales (RMB)

Number of Users Number of Transactions Sales (RMB)

RCT Sample Month Dummy

Panel A: Purchase Database

$0.893^{* * *}$
$(0.255)$

$\begin{array}{cc}-4.671 * * * & -1,565^{* * *} \\ (0.818) & (451.5)\end{array}$

(451.5)

\begin{tabular}{ccc}
$0.568 * *$ & $-5.290 * * *$ & -585.9 \\
$(0.274)$ & $(0.863)$ & $(458.0)$ \\
& & \\
$\checkmark$ & $\checkmark$ & $\checkmark$ \\
$\checkmark$ & $\checkmark$ & $\checkmark$ \\
100,098 & 100,098 & 100,098 \\
0.679 & 0.667 & 0.227 \\
8,471 & 8,471 & 8,471 \\
\hline
\end{tabular}

Village Fixed Effects

Control for Months Since Program Entry

Observations

R-squared

$(0.255)$

$(0.818)$

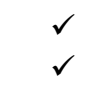

Number of Village Clusters

\begin{tabular}{cccc}
$\checkmark$ & $\checkmark$ & $\checkmark$ & $\checkmark$ \\
$\checkmark$ & $\checkmark$ & $\checkmark$ & $\checkmark$ \\
125,204 & 125,204 & 125,204 & 100,098 \\
0.694 & 0.68 & 0.219 & 0.679 \\
11,731 & 11,731 & 11,731 & 8,471 \\
\hline
\end{tabular}

(7) Full Sample

(8) Number of Transactions

(9)

3 Provinces

Dependent Variables:

Weight $(\mathrm{kg})$

Number of Transactions

Weight $(\mathrm{kg})$

RCT Sample Month Dummy

$-0.387 * * *$

$(0.0225)$

Village Fixed Effects

Control for Months Since Program Entry

Observations

R-squared

Number of Village Clusters
Panel B: Out-Shipment Database

$\begin{array}{ccc}-1.256 * * * & -0.498 * * * & -1.407 * * * \\ (0.125) & (0.0261) & (0.138)\end{array}$

(0.125)

$(0.0261)$

$(0.138)$

$\begin{array}{cccc}\checkmark & \checkmark & \checkmark & \checkmark \\ \checkmark & \checkmark & \checkmark & \checkmark \\ 120,483 & 120,483 & 95,744 & 95,744 \\ 0.592 & 0.432 & 0.57 & 0.422 \\ 11,904 & 11,904 & 8,591 & 8,591\end{array}$

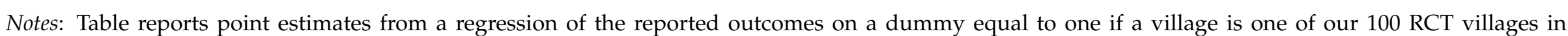

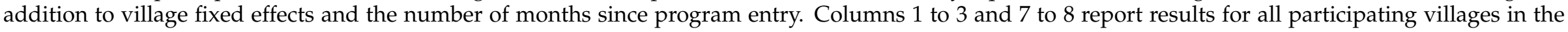

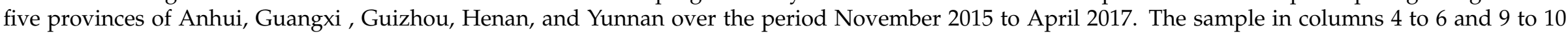

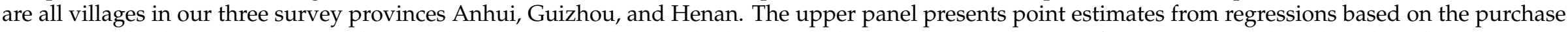

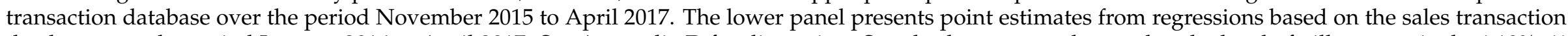

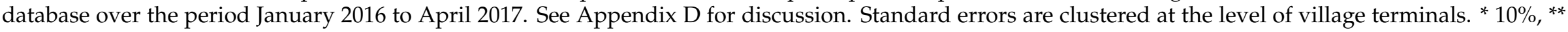
$5 \%$, *** $1 \%$ significance levels. 
Table A.14: Quantification Using Alternative Demand Parameters

\begin{tabular}{|c|c|c|c|c|c|c|c|c|c|}
\hline & \multicolumn{3}{|c|}{$\sigma_{-} \mathrm{D}=2.87, \sigma_{-} \mathrm{N}=2.85$} & \multicolumn{3}{|c|}{$\sigma_{-} \mathrm{D}=3.87, \sigma_{-} \mathrm{N}=3.85$} & \multicolumn{3}{|c|}{$\sigma_{-} \mathrm{D}=4.87, \sigma_{-} \mathrm{N}=4.85$} \\
\hline & $\begin{array}{c}\text { Durables } \\
\text { Consumption }\end{array}$ & $\begin{array}{l}\text { Non-Durables } \\
\text { Consumption }\end{array}$ & $\begin{array}{l}\text { Total Retail } \\
\text { Consumption }\end{array}$ & $\begin{array}{c}\text { Durables } \\
\text { Consumption }\end{array}$ & $\begin{array}{l}\text { Non-Durables } \\
\text { Consumption }\end{array}$ & $\begin{array}{l}\text { Total Retail } \\
\text { Consumption }\end{array}$ & $\begin{array}{c}\text { Durables } \\
\text { Consumption }\end{array}$ & $\begin{array}{l}\text { Non-Durables } \\
\text { Consumption }\end{array}$ & $\begin{array}{l}\text { Total Retail } \\
\text { Consumption }\end{array}$ \\
\hline $\begin{array}{l}\text { Reduction in Retail Cost of } \\
\text { Living for All Households }\end{array}$ & $\begin{array}{l}5.256 \% \\
(0.048)\end{array}$ & $\begin{array}{l}0.739 \% \\
(0.005)\end{array}$ & $\begin{array}{l}1.27 \% \\
(0.007)\end{array}$ & $\begin{array}{l}3.379 \% \\
(0.03)\end{array}$ & $\begin{array}{l}0.481 \% \\
(0.003)\end{array}$ & $\begin{array}{l}0.824 \% \\
(0.005)\end{array}$ & $\begin{array}{l}2.489 \% \\
(0.022)\end{array}$ & $\begin{array}{l}0.357 \% \\
(0.003)\end{array}$ & $\begin{array}{l}0.61 \% \\
(0.003)\end{array}$ \\
\hline $\begin{array}{l}\text { Reduction in Retail Cost of } \\
\text { Living Among Users }\end{array}$ & $\begin{array}{l}32.416 \% \\
(0.378)\end{array}$ & $\begin{array}{l}5.904 \% \\
(0.044)\end{array}$ & $\begin{array}{l}8.735 \% \\
(0.054)\end{array}$ & $\begin{array}{l}19.884 \% \\
(0.221)\end{array}$ & $\begin{array}{l}3.806 \% \\
(0.028)\end{array}$ & $\begin{array}{l}5.597 \% \\
(0.034)\end{array}$ & $\begin{array}{c}14.331 \% \\
(0.155)\end{array}$ & $\begin{array}{l}2.808 \% \\
(0.021)\end{array}$ & $\begin{array}{l}4.117 \% \\
(0.025)\end{array}$ \\
\hline
\end{tabular}

Notes: Table reports average household gains in terms of percentage point reductions in household retail cost of living across alternative parameterizations of household demand. Estimates are based on equation (3) using treatment effects on household substitution into new e-commerce option. See Evaluation Section for discussion. Standard errors are bootstrapped across 1000 iterations. 
Table A.15: Test for Effects on Attrition and Migration

\begin{tabular}{|c|c|c|c|c|}
\hline Dependent Variables & & Intent to Treat & $\begin{array}{c}\text { Treatment on } \\
\text { Treated } \\
\end{array}$ & $\begin{array}{c}\text { Log Distance } \\
\text { (IV using Treat) }\end{array}$ \\
\hline \multirow{4}{*}{ Attrition $(\mathrm{Yes}=1)$} & Treat or Log Dist & $\begin{array}{c}0.0138 \\
(0.0239)\end{array}$ & $\begin{array}{c}0.0258 \\
(0.0445)\end{array}$ & $\begin{array}{l}-0.00740 \\
(0.0127)\end{array}$ \\
\hline & R-Squared & 0.000 & & \\
\hline & Number of Obs & 2,629 & 2,629 & 2,629 \\
\hline & First Stage F-Stat & & 44.24 & 35.90 \\
\hline \multirow{4}{*}{$\begin{array}{l}\text { Number of Household } \\
\text { Members Who Moved } \\
\text { Back to the Village }\end{array}$} & Treat or Log Dist & $\begin{array}{c}0.0255 \\
(0.0400)\end{array}$ & $\begin{array}{c}0.0472 \\
(0.0734)\end{array}$ & $\begin{array}{l}-0.0129 \\
(0.0199)\end{array}$ \\
\hline & R-Squared & 0.001 & & \\
\hline & Number of Obs & 3,526 & 3,526 & 3,526 \\
\hline & First Stage F-Stat & & 45.27 & 42.71 \\
\hline \multirow{4}{*}{$\begin{array}{l}\text { Number of Household } \\
\text { Members Who Moved } \\
\text { Away from the Village }\end{array}$} & Treat or Log Dist & $\begin{array}{l}-0.00345 \\
(0.0184)\end{array}$ & $\begin{array}{l}-0.00637 \\
(0.0338)\end{array}$ & $\begin{array}{c}0.00174 \\
(0.00922)\end{array}$ \\
\hline & R-Squared & 0.012 & & \\
\hline & Number of Obs & 3,523 & 3,523 & 3,523 \\
\hline & First Stage F-Stat & & 45.44 & 43.84 \\
\hline \multirow{4}{*}{$\begin{array}{l}\text { Would You Be Willing to } \\
\text { Migrate to a City If a } \\
\text { Good Job Opportunity } \\
\text { Presented Itself? (Yes=1) }\end{array}$} & Treat or Log Dist & $\begin{array}{l}-0.0249 \\
(00191)\end{array}$ & $\begin{array}{l}-0.0458 \\
(00348)\end{array}$ & $\begin{array}{c}0.0125 \\
(000953)\end{array}$ \\
\hline & R-Squared & 0.025 & $(0.0540)$ & (0.00953) \\
\hline & Number of Obs & 3,527 & 3,527 & 3,527 \\
\hline & First Stage F-Stat & & 45.76 & 44.15 \\
\hline
\end{tabular}

Notes: Table reports point estimates from specification (1). The first column reports ITT and the second column TOT. The third column replaces the binary TOT with log residential distances to the nearest e-commerce terminal (using village-level ITT as instrument as for second column). See Appendix F for discussion. Standard errors are clustered at the level of villages. ${ }^{*} 10 \%,{ }^{* *}$ $5 \%,{ }^{* * *} 1 \%$ significance levels.

\section{Appendix B: K-L-K Indices}

Table 1 reports treatment effects after combining several outcomes related to consumption, incomes and local retail prices into three indices. We follow Kling et al. (2007) ("K-L-K") and construct equally weighted averages of z-scores that we compute by subtracting outcomes by the mean of the variable in the control group and dividing by the standard deviation of the variable in the control group. The z-scores are signed such that effects on all index components point in the same direction (i.e. price index reductions or income growth). If a household (or store) has a valid response to at least one component measure of an index, then any missing values for other component measures are imputed at the random assignment group mean. This results in differences between treatment and control means of an index being the same as the average of treatment and control means of the components of that index, so that the index can be interpreted as the average of results for separate measures scaled to standard deviation units. ${ }^{1}$

\footnotetext{
${ }^{1}$ For two outcomes of the consumption index discussed below, the control mean and standard deviation were zero. In those cases, we instead use the standard deviation of the variable observed in the full sample.
} 
The consumption index is based on 11 variables related to household substitution of expenditures into the new e-commerce shopping option, all entering the index positively. Those outcomes are whether a household reports ever having used the new option, reported usage over the past month, and the shares of household total retail expenditure spent on 9 consumption categories (food and beverages, tobacco and alcohol, medicine and health, clothing and accessories, other every-day products, fuel and gas, furniture and appliances, electronics, transport equipment). The treatment effects on each of these outcomes are reported as part of appendix Table A.6.

The income index is based on 14 variables related to income generation, labor supply, online selling activity and online sourcing of inputs. Those outcomes are monthly income per capita, annual income per capita, monthly income from agriculture, monthly income from non-agriculture, monthly hours of work by primary earner, monthly hours of work by secondary earner, whether anyone in household has ever sold online, sold over the last month, revenues from online sales over past month, share of online revenues in total monthly income, whether primary earner is a farmer (entering negatively), whether any household member has started a new business over past 6 months, whether the new business sells in part online, and the share of monthly online purchases in total expenditures on inputs and materials. The treatment effects on each of these outcomes are reported as part of appendix Table A.7.

The local retail index is based on 4 store-level measures related to effects on the local retail cost of living. Those outcomes are the average of log price changes of continuing product items within the store (entering negatively), the number of new product additions over the past month (positively), the number of product replacements (measured as the fraction of products reported in the baseline survey that were no longer available at endline) (negatively), and whether or not the store owner reports sourcing products online (positively). The treatment effects on each of these outcomes are reported as part of appendix Table A.8.

\section{Appendix C: Role of Spillovers}

To investigate the role of spillovers, we pursue two different approaches. First, we follow an approach similar to Miguel \& Kremer (2004):

$$
y_{h v}^{\text {Post }}=\alpha+\beta_{1} \text { Treat }_{v}+\beta_{2} \text { Exposure }_{v}^{\text {treat }}+\beta_{3} \text { Exposure }_{v}^{\text {all }}+\gamma y_{h v}^{\text {Pre }}+\epsilon_{h v},
$$

where Exposure $v_{v k}^{\text {treat }}$ measures the proximity of village $v$ to other program villages, and Exposure ${ }_{v k}^{a l l}$ measures proximity to all villages on the candidate list from which we randomly selected our control villages. Even though exposure to other program villages is not randomly assigned, our randomization means that conditional on exposure to all candidate villages, exposure to other treatment villages is plausibly exogenous. Using this design, $\beta_{2}$ is an estimate of the the strength of cross-village spillovers. We measure exposure as the number of intent-to-treat villages within 3 or $10 \mathrm{~km}$ distance bins of a given village. Table A.10 reports the estimation results. We find some evidence of positive spillover effects of nearby terminals within $3 \mathrm{~km}$ of the village. These effects imply a larger total average effect on e-commerce uptake. Consumption uptake increases from 9 percent in Table A.6 to 14 percent once we take into account positive spillovers from nearby villages, which is 13 percent of the village population after adjusting for sampling weights. In contrast, we find no evidence of cross-village spillovers on local retail stores, or on the production 
side of the economy.

Second, to further investigate these channels in the absence of experimental variation in program saturation rates, ${ }^{2}$ we also pursue an approach grounded in trade theory. In particular, we can quantify the fraction of a rural location's total trade market access that is due to trading exposure to other rural markets in the same county. This fraction provides additional information on the extent of rural-to-rural spillovers from other sample villages in our setting. If a sizable share of local market access is due to trading relations with other local rural markets, then indirect effects on local product prices and incomes from treatments in other villages could become an important force. If, on the other hand, local product and factor prices are predominantly determined by access to larger urban markets, then rural-to-rural spillovers could have negligible effects on local prices and incomes across our sample villages.

Following e.g. Head \& Mayer (2014), the market access of location $v$ to all other rural and urban markets $j \neq v$ is:

$$
M A_{v}=\sum_{j \neq v} \tau_{j v}^{-\theta} Y_{j}
$$

where $\tau_{j v}$ is the bilateral trade cost, $\theta$ is the elasticity of trade flows with respect to trade costs, and $Y_{j}$ is a measure of $j^{\prime}$ s market size. ${ }^{3} M A_{v}$ is thus a weighted sum of economic activity outside of market $v$, with weights that are inversely related to bilateral trade costs. To compute the fraction of total market access that is due to bilateral linkages with other rural markets in the same county (i.e. $M A_{v}^{R} / M A_{v}$ ), we compute (6) both across bilateral connections to all other markets (denominator), and only summing across bilateral connections with other rural markets in the same county (numerator). Alternatively, we restrict the numerator to bilateral connections with respect to the fraction of rural markets in the county that are participating in the program to compute the share of market access due to rural locations with program terminals. That fraction was about $1 / 6$ th of all rural markets in participating counties over our sample period.

To compute these measures, we use the township-level data from the Chinese Population Census in 2010 described in Appendix F below. These data provide us with the populations residing in each of roughly 45,000 township-level administrative units. In addition, we use the coordinates of township centroids to construct the full matrix of bilateral distances in $\mathrm{km}$. Following the trade literature, we use these bilateral distances to parameterize $\tau_{j v}^{-\theta}:$ using the finding that the elasticity of trade flows with respect to distance is approximately $-1{ }^{4}$ we measure $\tau_{j v}^{-\theta}$ as the inverse bilateral distance in $\mathrm{km}$ when summing across the $j$ market sizes. Alternatively, we also use a larger distance elasticity of -1.5 that gives more weight to markets in closer proximity. For market size $Y_{j}$, we use either population or population multiplied by the value added per worker for rural and non-rural workers measured at the province level for 2010. The first met-

\footnotetext{
${ }^{2}$ As part of our negotiations and collaboration with the firm's local implementation teams, it was not feasible to also attempt a two-stage cluster randomization design that would have allowed us to randomly vary saturation rates.

${ }^{3}$ To be consistent with structural gravity in trade models, the measure $Y_{j}$ of $j$ 's market size should include a multilateral resistance term capturing $j$ 's own degree of access to all other markets (see e.g. Head \& Mayer (2014)). In (A.2), we abstract from this and compute a first-order approximation of the structural gravity expression for $M A_{v}$. In practice, both measures have been found to yield very similar results in recent empirical work, as they are highly correlated (e.g. Donaldson \& Hornbeck (2016)).

${ }^{4}$ See e.g. Disdier \& Head (2008) for a meta-analysis of this point estimate.
} 
ric provides an inverse distance-weighted measure of market access to populations outside the township, while the second provides an approximate measure of access to GDP. Finally, we define rural and urban markets following the administrative classification across township-level units we obtain in the census data. For computational feasibility, when constructing the full matrix of bilateral connections, we compute the total market access of rural townships with respect to all other township units (both rural and urban) within each of the 3 broad administrative regions of China in which our sample counties are located: East China (7 provinces), Middle China (3 provinces) and Southwest China (5 provinces). ${ }^{5}$

The above provides us with four measures of the ratio of total market access that is due to access to other rural populations or rural GDP within the the same county: measured either in terms of access to population or to GDP, and measured either in terms of access to all rural markets in the county or only the fraction of rural markets that on average participate in the e-commerce program. We compute the median, mean and standard deviations of these 4 ratios for all rural townships located in the three regions of China, as well as only for townships in our 3 sample provinces, or only for townships in the 8 sample counties. Furthermore, we compute each of these measures both for the baseline distance elasticity of -1 , and when using -1.5 instead.

Appendix Table A.11 presents the estimation results. Overall, we find that other rural markets in the same county account for a tiny fraction of total trade market access for the median or the average rural market place. This result is driven by the fact that nearby rural markets within the same county account for a small fraction of the market size that is concentrated in vastly larger urban centers. This is particularly the case when using economic output as the measure of market size, but also holds for raw populations. For example, the median fraction of market access from nearby rural markets in terms of GDP is 0.37 percent in our sample provinces, and 1.2 percent in terms of population access. These fractions slightly increase when giving more weight to nearby markets using a higher distance elasticity, but remain close to zero in both cases when computing rural-to-rural market access only with respect to the average fraction of rural markets that are participating in the program in any given county over our sample period. These findings are in line with the absence of significant GE spillover effects on market prices or nominal incomes shown in our first approach above, and serve to provide some further corroborating evidence in this context.

\section{Appendix D: Additional Results from the Firm's Database}

\section{Are the RCT Sample Villages Representative?}

One concern is that the 8 counties that our RCT takes place in may not be representative of program villages in the Chinese countryside more broadly. To assess whether the RCT villages are representative of the population of program villages in China, we use the 5-province transaction database on both purchases and sales transactions to estimate regressions of the following form:

$$
y_{v m}=\theta_{m}+\beta \text { RCTSample }_{v}+\gamma \text { MonthsSinceEntry }_{v m}+\epsilon_{v m}
$$

\footnotetext{
${ }^{5}$ The 8 counties of our RCT fall into one these three zones. Omitting regions outside each zone is somewhat conservative, as their inclusion would increase the denominator of the rural-to-total market access ratios.
} 
where $v$ indexes villages and $\theta_{m}$ is a set of monthly dummies indexed by $m$ for the 18 months of operation from November 2015 to January 2017. $y_{v m}$ is one of five village-level monthly outcomes (number of buyers, number of purchase transactions, total terminal sales, number of outshipments and total weight of out-shipments in $\mathrm{kg}$ ), RCTSample is a dummy for whether the village is in our RCT sample, and MonthsSinceEntry controls for the number of month that the program has been in operation in $v$ as of month $m$. The standard errors $\epsilon_{v m}$ are clustered at the village level.

The results in appendix Table A.12 show no remarkable differences between our RCT villages and the population of program villages in these 5 provinces. The same is true if we compare our RCT villages to all villages in our 3 survey provinces. The RCT sample seems marginally more successful on the out-shipment side, but the magnitudes are tiny. These results provide some reassurance against the potential concern that the e-commerce firm directed our team towards 8 counties that systematically differ from the program's target locations in the Chinese countryside.

\section{Did We Collect Endline Data During Particular Months?}

The timeline of pre-treatment data collection was determined by the roll-out schedule of the e-commerce firm, and we could not finance more than a single post-treatment round. As a result of these constraints, our survey cannot measure the impact of seasonality on treatment effects. We therefore use the transaction database to study seasonality effects by estimating:

$$
y_{v m}=\theta_{v}+\beta R C \text { TMonth }{ }_{m}+\gamma \text { MonthSinceEntry } y_{v m}+\epsilon_{v m},
$$

where RCTMonth is a dummy for our survey months i.e., a dummy equal to 1 if month $m$ is either in December, January, April or May, which are the four calendar months during which we conducted our survey. We again cluster standard errors $\epsilon_{v m}$ at the village level. The results are in appendix Table A.13. We find slightly higher numbers of buyers during survey months relative to the rest of the calendar year, and slightly lower numbers of purchase transactions and out-shipments. In both cases, the point estimates are very small: about one additional buyer per month, a reduction of between 4 to 5 in the number of monthly purchase transactions, and a reduction of less than one out-shipment per month on the selling side. We conclude that seasonality is unlikely to be a significant driver underlying the findings of the RCT.

\section{Appendix E: Welfare Evaluation}

Following recent work by Atkin et al. (2018), we propose a three-tier demand system to describe household retail consumption across product groups, retail shopping options and products. In the upper tier, shown in equation A.3, there are Cobb-Douglas preferences over broad product groups $g \in G$ (durables and non-durables) in total consumption. In the middle tier, shown in equation A.4, there are asymmetric CES preferences over local retailers selling that product group $s \in S$ (e.g. local stores, market stalls or the e-commerce option). In the final tier, there are preferences over the individual products within the product groups $b \in B_{g}$ that we can 
leave unspecified for now.

$$
\begin{aligned}
U_{h} & =\prod_{g \in G}\left[Q_{g h}\right]^{\alpha_{g h}} \\
Q_{g h} & =\left(\sum_{s \in S_{g}} \beta_{g s h} q_{g s h}^{\frac{\sigma_{g}-1}{\sigma_{g}}}\right)^{\frac{\sigma_{g}}{\sigma_{g}-1}},
\end{aligned}
$$

where $\alpha_{g h}$ and $\beta_{g s h}$ are (potentially household group-specific) preference parameters that are fixed across periods. $Q_{g h}$ and $q_{g s h}$ are product-group and store-product-group consumption aggregates with associated price indices $P_{g h}$ and $r_{g s h}$ respectively, and $\sigma_{g}$ is the elasticity of substitution across local retail outlets. For each broad product group, consumers choose how much they are going to spend at different retail outlets based on the store-level price index $r_{g s h}$ (which itself depends on the product mix and product-level prices on offer across outlets).

While the demand system is homothetic, we capture potential heterogeneity across the income distribution by allowing households of different incomes to differ in their expenditure shares across product groups $\left(\alpha_{g h}\right)$ and their preferences for consumption bundles at different stores within those product groups $\left(\beta_{g s h}\right.$ and the preference parameters that generate $\left.q_{g s h}\right)$. As shown by Anderson et al. (1992), these preferences can generate the same demands as would be obtained from aggregating many consumers who make discrete choices over which store to shop in. Building on Feenstra (1994), the following expression provides the exact proportional cost of living effect (CLE) under this demand system as a fraction of initial household expenditures:

$$
\frac{C L E}{e\left(\mathbf{P}^{0}, u_{h}^{0}\right)} \prod_{g \in G}\left(\left(\frac{\sum_{s \in S_{g}^{C}} \phi_{g s h}^{1}}{\sum_{s \in S_{g}^{C}} \phi_{g s h}^{0}}\right)^{\frac{1}{\sigma_{g}-1}} \prod_{s \in S_{g}^{C}}\left(\frac{r_{g s h}^{1}}{r_{g s h}^{0}}\right)^{\omega_{g s h}}\right)^{\alpha_{g h}}-1
$$

where $S_{g}^{C}$ denotes the set of continuing local retailers within product group $g, \phi_{g s h}^{t}=$ $r_{g s h}^{t} q_{g s h}^{t} / \sum_{s \in S_{g}} r_{g s h}^{t} q_{g s h}^{t}$ is the expenditure share for a particular retailer of product group $g$, and the $\omega_{g s h}$ s are ideal log-change weights. ${ }^{6}$

For each product group $g$, the expression has two components. The $\prod_{s \in S_{g}^{C}}\left(\frac{r_{g s h}^{1}}{r_{g s h}^{0}}\right)^{\omega_{g s h}}$ term is a Sato-Vartia (i.e. CES) price-index for price changes in continuing local stores that forms the pro-competitive price effect. ${ }^{7}$ The price terms $r_{g s h}^{t}$ are themselves price indices of product-specific prices $p_{g s b}^{t}$ within local continuing stores which, in principle, could also account for new product varieties or exiting product varieties using the same methodology. While we name these price changes pro-competitive, they may derive from either reductions in markups or increases in productivity at local stores (distinctions that do not matter on the cost-of-living side, but would generate different magnitudes of profit and income effects that we capture on the nominal income side).

The $\left(\frac{\sum_{s \in S_{g}^{C}} \phi_{g s h}^{1}}{\sum_{s \in S_{g}^{C}} \phi_{g s h}^{0}}\right)^{\frac{1}{\sigma_{g h}-1}}$ term captures the gains to customers of the e-commerce option in the nu-

\footnotetext{
${ }^{6}$ In particular, $\omega_{g s h}=\left(\frac{\tilde{\phi}_{g s h}^{1}-\tilde{\phi}_{g s h}^{0}}{\ln \tilde{\phi}_{g s h}^{1}-\ln \tilde{\phi}_{g s h}^{0}}\right) / \sum_{s \in S_{g}^{C}}\left(\frac{\tilde{\phi}_{g s h}^{1}-\tilde{\phi}_{g s h}^{0}}{\ln \tilde{\phi}_{g s h}^{1}-\ln \tilde{\phi}_{g s h}^{0}}\right)$, which in turn contain expenditure shares of different retailers within product groups, where the shares consider only expenditure at continuing retailers $\tilde{\phi}_{g s h}^{t}=r_{g s h}^{t} q_{g s h}^{t} / \sum_{s \in S_{g}^{c}} r_{g s h}^{t} q_{g s h}^{t}$

${ }^{7}$ Notice that the assumption of CES preferences does not imply the absence of pro-competitive effects as we do not impose additional assumptions about market structure (e.g. monopolistic competition).
} 
merator, from both a direct price index effect due to the new shopping option and potential other local store entry induced by this change, and local store exit in the denominator, i.e. the exit effect.

Now consider the case-as in the final section of the paper-where the program's effect on cost of living is driven by the direct price index effect. In that case, the expenditure share spent on continuing local retailers $\left(\sum_{s \in S_{g}^{C}} \phi_{g s h}^{1}\right)$ is lower than unity only due to substitution into the new e-commerce option. The consumer gains from the program as a proportion of initial household spending are then:

$$
\frac{C L E}{e\left(\mathbf{P}^{0}, u_{h}^{0}\right)}=\prod_{g \in G}\left(\left(\sum_{s \in S_{g}^{C}} \phi_{g s h}^{1}\right)^{\frac{1}{\sigma_{g}-1}}\right)^{\alpha_{g h}}-1 .
$$

The welfare gain from a new shopping option is a function of the market share of that outlet post-entry and the elasticity of substitution across stores. The revealed preference nature of this approach is clear. If consumers greatly value the arrival of the new option-be it because it offers low prices $p_{g s b}^{1}$, more product variety that reduces $r_{g s h}^{1}$ or better amenities $\beta_{g s h}$-the market share is higher and the welfare gain greater. Hence, these market share changes capture all the potential consumer benefits of shopping through the e-commerce option. The magnitude of the welfare gain depends on the elasticity of substitution. Observed e-commerce market shares will imply smaller welfare changes if consumers substitute between local shopping options very elastically, and larger welfare changes if they are inelastic. A similar logic would apply to effects on the entry of local retailers, or on the exit of local stores (where a large period 0 market share means large welfare losses, again tempered by the elasticity of substitution).

\section{Appendix F: RCT and Data Appendix}

\section{F.1 Program Description and Background}

Following the announcement of the policy objective to expand e-commerce to the Chinese countryside as part of the so-called Number One Central Document in January 2014, the Chinese government entered a partnership with a large firm that operates a popular Chinese e-commerce platform. The program's objective is to provide e-commerce access in rural markets at the same price, convenience and service quality that buyers and producers face in their county's main city center. The firm's objective as part of the program is to penetrate the vast and largely untapped e-commerce market outside of Chinese cities. Rural expansion is one of the firm's strategic priorities over the coming years.

The program makes two main types of investments to enable villagers to buy and sell online through the firm's platform. First, the program invests in the local distribution network, which the firms views as a necessary condition to provide e-commerce access in rural areas. Before the arrival of the program, most villages were not serviced by commercial parcel delivery operators, who had not solved the problem of the "last mile" transportation between dispersed rural households and urban county centers. ${ }^{8}$

The program sets out to change this lack of service with logistics investments targeted at e-commerce. In particular, the firm oversees the construction of warehouses that serve as logistical nodes to pool all e-commerce-related transportation requests to and from the participating

\footnotetext{
${ }^{8}$ To receive packages via mail in absence of commercial parcel delivery services, rural households have to travel to the county or township center to pick up the package after receiving notification by mail that it has arrived.
} 
villages. These warehouses are located close to the main urban center of the counties with good cross-county transport access. The program also fully subsidizes the transportation cost between these warehouses and participating villages, so that rural households face the same delivery costs and prices as households in the urban parts of the county. The rationale for this subsidy is that village deliveries and pickups start from a low basis, which due to economies of scale in rural transportation makes the starting phase of e-commerce prohibitively costly for village customers despite the investments in warehouses. The calculation of the government and the firm is that as the scale of rural e-commerce grows, per unit transport costs will decline enough to remove the need for a subsidy. Neither the warehouses nor the last-mile subsidy can be used for shipments outside of the firm's e-commerce platform.

The second investment is the installation of a program terminal in a central village location. The e-commerce terminal is a PC, keyboard and mouse connected to a flat-screen monitor mounted on the wall of a dedicated shop space and displaying the firm's website. On the screen, consumers and producers can choose their purchases or see their sales requests on the platform. The firm employs a terminal manager to assist local households in buying and selling products through the firm's e-commerce platform. The terminal manager receives a reward of about 3-5 percent for each transaction completed through the terminal. Before deciding on terminal installations, the firm solicits applications from potential local store operators and schedules an exam for the applicants. The score of this exam is one of the criteria that the firm uses to determine whether a village is a candidate. Villagers can pay in cash when the products arrive at the store for pickup, or they get paid upon delivery of their products for pickup at the store location if selling online. Instead of using the terminal interface, households can also use the firm's e-commerce platform remotely on smartphones or PCs to order product deliveries or pickups at the terminal location. When referring to the new e-commerce option in the text, we include all types of use of the e-commerce platform. The firm views the option to use the village terminals as overcoming three challenges that are specific to the rural population. First, local households may not be used to or comfortable with navigating online platforms. Second, they often do not have access to online payment methods. And third, they may not trust online purchases or sales before inspecting the goods in person or having interacted with buyers directly.

\section{F.2 Surveyor Training and Quality Management}

Piloting and Surveyor Training Our survey supervisors are professionals from the Research Center for Contemporary China (RCCC) at Peking University. All RCCC supervisors have previous experience conducting large scale surveys in rural China. Before each of the two survey rounds, we traveled to Beijing to lead a one-day training workshop targeted at the supervisors and a group of graduate students from Renmin University and Jinan University, who were working with us as research assistants on this project. This training walked the RCCC supervisors and our graduate students through each step of the survey design, data collection protocols and quality control protocols that we had shared with them to study carefully in advance. Given budget and time constraints, the survey was paper based. Prior to our baseline survey, RCCC supervisors and our team of graduate students tested our survey design in a pilot survey of 45 households in two villages located in the rural parts of Hebei Province.

In the field, each supervisor was in charge of a team of six surveyors. In addition to the 
supervisors, two of our trained graduate students accompanied each team in the field. The role of the graduate students was to both support and monitor the recruitment and training of the local surveyors and the data collection, and to report back to us with detailed daily progress reports. Given differences in local dialects and rural conditions, the RCCC recruited surveyors among local university students from the provinces in which the data collection took place. All surveyors were familiar with the local dialect and customs of the rural areas in their home province. Each surveyor completed at least two full days of training and supervised practice questionnaire interviews before joining our field survey team. As part of the training, we provided surveyors with a number of supporting documents. In particular, they received an example of a completed representative survey questionnaire, detailed instructions on how to assist households in answering the questionnaire, a set of cards containing descriptions and examples of consumption products within categories or income-generating activities within sectors, and a set of solutions and best practices for common survey challenges. As described in Appendix F.5 below, we also trained surveyors to use separate pre-prepared spreadsheets to list individual household purchase transactions within product categories or income flows by type of activity. These spreadsheets were used for households to list individual transactions over a given period of time and within categories, before aggregating this information up to complete the final survey questionnaire cells. As part of their training, surveyors were trained to double-check with respondents any answer to the questionnaire that appears inconsistent with a previous answer.

Data Quality Management and Cleaning Surveyors conducted the household survey in teams of two. During the interview, surveyors completed the questionnaire, along with supporting documents used to help households recall, categorize and sum up their consumption expenditures or earnings (we further describe data collection and variable construction for expenditure and earning variables below). As part of quality control, supervisors reviewed one randomly chosen completed questionnaire, supporting documents, and interview audio tape from each surveyor at the end of every day. ${ }^{9}$ In addition, our graduate students monitored the survey teams by accompanying them for part of their interviews, and reported back to the supervisors and our team in case of concerns. During recruiting and surveyor training, the surveyors had been informed that lack of accuracy, diligence or patience in the interviews would lead to the termination of employment, while a good record guaranteed a letter of recommendation confirming participation in our research project.

We also asked our surveyors to rate each household respondent along a number of dimensions such as cooperativeness, reliability, level of understanding, and level of interest in our survey. Surveyors also recorded the presence of any other household or non-household member whose presence could affect answers to our questionnaire. In our analysis of the data, we paid special attention to the reliability rating: 1. completely reliable, 2. mostly reliable, and 3. sometimes not reliable. Whenever surveyors rated a respondent as "sometimes not reliable", they also wrote down an explanation for this rating. On the basis of these written explanations, we created a clean household survey dataset. This dataset excludes 0.25 percent of unreliable/uncooperative households entirely from the sample. In other cases, surveyors' explanation suggested that only answers to a particular section of our questionnaire were

\footnotetext{
${ }^{9}$ Some households opted out of audio-recording.
} 
unreliable. Using this information, we set all income variables to missing for 1.06 percent of all household respondents, all consumption variables to missing for 0.4 percent of households, and all income and consumption variables to missing for 1.31 percent of households. The descriptive statistics in Tables A.1 to A.4 are based on this cleaned household survey dataset. When using total nominal retail expenditure or incomes in RMB as part of the dependent variables on the left-hand side of the regressions, we censor these reported values at the one-percent level from the left and right tails within the survey round. ${ }^{10}$ Similarly, price changes between rounds are censored at the 1 percent level. The point estimates remain statistical zeros in all these cases, as is the case post-censoring in the draft, but the standard errors slightly increase. Appendix F.5 below provides additional information about variable construction.

\section{F.3 Experimental Design}

Appendix Figure A.1 presents a map of the locations where the RCT takes place. Tables A.1 to A.4 present descriptive statistics.

\section{Selection of Provinces and Counties}

There are two main factors determining our survey location in Anhui, Henan and Guizhou, and the 8 counties within these provinces. First, our survey location depended on the timing of the program's roll-out across different provinces and counties, which had been decided before our collaboration with the firm. Second, we were guided by the internal evaluation of the program's senior managers as to whether the provincial and county managers in question would be willing to cooperate with our research protocol. These counties are: Huoqiu (Anhui), Linying (Henan), Linzhou (Henan), Minquan (Henan), Suixi (Anhui), Tianchang (Anhui), Xifeng (Guizhou) and Zhenning (Guizhou). In Appendix D, we are also able to investigate the representativeness of our sample villages relative to all participating villages using the firm's internal transaction data in 5 provinces over this period.

\section{Selection of Villages and Randomization}

The unit of randomization is the village. For each county, we obtain a list of candidates that had been extended by 5 promising village candidates that would have not been part of the list in absence of our research. The three main factors determining the village selection within a county from the firm's operational perspective are i) a sufficient level of local population, ii) accessibility by roads, and iii) the presence of a capable store applicant (as measured by the applicant's test score). Overall, we are able to implement randomization on a broad pool of villages selected for participation in the program. This pool, however, is not a random sample of China' rural areas, but instead is likely a group of villages positively selected within each county, with better expected conditions for e-commerce usage on both consumption and production sides.

Upon receipt of this extended list of village candidates for each county, we randomly select 5 control villages and 7-8 treatment villages. The remaining villages on the extended list receive program terminals as planned. The full sample thus includes 40 control villages and 60 treatment villages across the 8 counties, which we selected from a total number of candidates of 432 villages that we received in the extended listings from the 8 county operations teams (on average 54

\footnotetext{
${ }^{10}$ Given more than one percent of observations report zero incomes, nominal incomes are only censored at the one-percent level from the right tail.
} 
villages per county). We restrict the list of villages entering the stratification and randomization to villages with at least $2.5 \mathrm{~km}$ distance to the nearest village on the county list, where possible. ${ }^{11}$ We then stratify treatment and control villages along four dimensions. First, we balance the selection of treatment and control to both have a ratio of 85:15 with respect to pre-existing availability of commercial package delivery ( $85 \%$ not available, $15 \%$ available), which is close to the observed ratio among all candidate villages. We obtain information on the availability of commercial package delivery for each village on the candidate list from the program's local county managers (who are not aware what we require that piece of information for). As we discuss below, having villages in our sample with pre-existing commercial delivery services allows us to further investigate the effect of the program that is driven by the terminal access point (i.e. the effect of lifting only the transactional barrier), relative to the effect of providing both the terminal access point and the necessary logistics for local e-commerce deliveries and pick-ups (i.e. the effect of lifting both the transactional and logistical barrier to e-commerce). We further stratify the selection of treatment and control villages on the basis of the equally-weighted average of the z-scores for three village variables: the local store applicants' test score, the village population, and the ratio of non-agricultural employment over the local population. We obtain the last variable from the establishment-level data of the Chinese Economic Census of 2008 which surveys every non-agricultural establishment in the counties.

\section{Sampling of Households, Response Rates and Attrition}

Our team was granted a two-week window for data collection, after receiving the extended candidate list of candidate villages from the local operation team in each county. Given this tight timeline, we were unable to conduct a village census for sampling purposes. Instead, our survey teams created detailed maps of all residences in the village to implement a random walk procedure. ${ }^{12}$

From each village's map, we defined an "inner zone" of residences within a 300 meter radius of the planned terminal location and an "outer zone" outside that radius. In the baseline data collection (December 2015 and January 2016 in Anhui and Henan, and April and May 2016 in Guizhou), the objective was to sample 14 households from the inner zone and 14 households from the outer zone. To randomly sample households within these zones, we selected 24 residences in both inner and outer zones. The household sampling proceeds as follows: we first randomly assign numbers to all residences within the zone on the map from 1 to $n$, and then define a rounded integer number $n / 24$. Starting from household number 1 , we then collect survey data from every household number in steps of the integer $n / 24$ until we have completed 14 surveys within the zone. For the endline data collection (12 months after baseline in each village), we implement the same procedure for all households that were not part of the baseline

\footnotetext{
${ }^{11}$ In counties with relatively short candidate lists we had to marginally extent this threshold, leading to a small number of villages with less than $2.5 \mathrm{~km}$ distances to the nearest other villages on the candidate list. The mean and median distances for villages without terminals to the nearest terminal location were 10.6 and $9.1 \mathrm{~km}$ respectively. Also see related spillover analysis in Appendix C.

${ }^{12}$ We use the boundary of the "natural village" as opposed to the "administrative village". Both of these are known delineations in China. The natural village captures a geographically contiguous rural population. Administrative villages are units with a village committee. In some cases, the administrative village includes more than one natural village.
} 
survey to select 10 additional households within the inner zone. ${ }^{13}$ In the few cases in which there were fewer than 24 residences within the inner zone, we extended the radius until we obtain at least 24 residences on the map. If either the survey respondent or the primary earner of the initially surveyed household no longer resides at the same address, we record this in our data and replace the household with another randomly sampled household within the same sampling zone (inner or outer). In our welfare analysis, we report results both before and after weighting each sampled household in proportion to the share of the village population in its sampling zone.

After introducing our survey to households, our surveyors asked for the household member with the best knowledge of household consumption expenditures and household incomes to respond to the questionnaire. In case nobody answered the door, or in case this most suited household member was not at home during our surveyors' first visit, the surveyors returned at least twice to complete the interview, often outside of working hours. Surveyors were also instructed to skip households with a most knowledgeable respondent older than 75 . Overall, our surveyors found willing and able respondents in two thirds of visited residences (66.1 percent). ${ }^{14}$ In the endline, we sampled 10 additional households from the inner zone. We used the same sampling methodology as in the baseline. Given expected sample attrition and the objective of 10 randomly selected additional households, the survey teams created a list of 22 new residential addresses in the inner zone and 6 new addresses in the outer zone. In the endline, we replaced a household respondent from the baseline whenever either the household had moved, the primary earner was no longer living there or the original baseline respondent was unavailable after three interview attempts. Using this rule, 71 percent of baseline respondents completed our questionnaire in the endline. As documented in appendix Table A.15, this percentage does not differ in treatment and control villages.

\section{F.4 Retail Price Survey}

Store Sampling Prior to the field survey, RCCC supervisors performed a census of all retail stores and market stalls ("stores" for short) located in the village and within a 15-minute walking distance of the boundaries of the natural village. Most villages have fewer than five stores, so in most villages we sampled products from all stores and market stalls in the vicinity of the village. If there were more than 15 stores in a village, we instructed supervisors to collect a representative sample of local retail information, giving more weight (i.e. more price quotes) to more popular establishments within product groups.

Product Sampling and Data Collection The data collection for the local retail price survey was conducted by the trained RCCC supervisors. We aim to collect data on 115 price quotes for each village. 100 of these prices are from the same 9 household consumption categories for retail products as in our household survey (food and beverages, tobacco and alcohol, medicine and health, clothing and accessories, other every-day products, fuel and gas, furniture and appliances, electronics, transport equipment), and 15 price quotes are for local production and business inputs. Our protocol for the price data collection closely follows the IMF/ILO standards

\footnotetext{
${ }^{13}$ This extended sample was possible due to a small remaining positive balance on the project account that we decided to invest in expanding the household survey sample.

${ }^{14}$ Of the one third of addresses at which our surveyors did not encounter willing and able respondents, 56.6 percent had nobody at home during any of our three visits, 30.5 percent refused to participate in the survey, 7.5 percent had no qualified respondent (due to old age), and 5.4 percent had no one living there.
} 
for store price surveys that central banks collect to compute the CPI statistics. The sampling of products across consumption categories is based on budget shares of rural households in Anhui and Henan that we observe in the microdata of the China Family Panel Study (CFPS) for 2012. Reflecting these consumption weights, supervisors in the baseline survey data aim to collect 47/100 price quotes in food and beverages, 15/100 in tobacco and alcohol, 9/100 in medicine and health, 9/100 in clothing and accessories, 4/100 in other every-day products, 4/100 in fuel and gas, 4/100 in furniture and appliances, 4/100 in electronics and 4/100 in transport equipment. In addition, we collect 15 price quotes for purchases of inputs to production or businesses. ${ }^{15}$

We provided supervisors with pre-prepared price surveys reflecting the number of observations to be collected for each product group. As for the collection of data on household expenses that we discuss above and in Appendix F.5 below, the supervisors were provided with detailed product cards that list product groups within each of the 10 broad categories above, as well as examples of product types within those subgroups of products. They also received instructions on product sampling, for instance about how to evaluate the popularity of an individual product by measuring shelf space and recurrence across different stores. To ensure that we can match identical products in both survey rounds, supervisors saved a picture of each product and recorded product characteristics at the barcode-equivalent level, including packaging type, size, and a detailed product description (name, brand, flavor, etc) wherever possible. ${ }^{16}$ For 78 percent of products collected in the baseline, we were able to find the exact same product in the same store one year later in the endline. As documented in appendix Table A.8, this percentage is somewhat smaller in intent to treat villages than in control villages, but this difference is not statistically significant. One challenge of surveying prices in rural China is a frequent lack of price tags displayed in store. As shown in Table A.4, about two thirds of the surveyed products lacked a price tag. In these cases, supervisors asked the store owner for the price that villagers would pay for the product. As part of quality control, we asked supervisors to rate the reliability of store owners' price quotes as good, average or poor. None of the reported findings change in sign, size or statistical significance when limiting the sample to price quotes from labeled products only or excluding reportedly unreliable price quotes.

\section{F.5 Variable Construction}

To collect data on household consumption expenditures and incomes from different activities, we trained the surveyors in using separate pre-prepared spreadsheets before filling out the final survey questionnaires. For expenditures, there is one spreadsheet for each of the nine categories that we include in retail consumption, and a separate sheet for business inputs. This allowed households to recall and list all relevant expenses or income flows within a given product group or type of activity over a given period of time. This transaction-level information was then aggregated in the presence of the household to complete the final survey questionnaire sections on expenditures or income flows.

To help respondent recall and categorize their expenditures, surveyors also received cards

\footnotetext{
${ }^{15}$ Supervisors sometimes failed to find enough products in a given category within the village. This was often the case for the durable goods categories. In such cases, supervisors replaced products in these missing categories with additional price quotes for products in "other every-day products".

${ }^{16}$ Some store owners refused to let supervisors take pictures. In such cases, we identify identical products in the endline data based on the same store and the detailed recorded product description.
} 
with examples of products in each category. The product cards break down the retail consumption space into 169 product types within the 10 broad categories we list above. After recording each item in a given category, surveyors go through the list of items and ask respondents how much they paid for each listed purchase. In addition to allocating transactions to different consumption product groups, the surveyors also recorded the modality of each listed purchase transaction (e.g. online vs offline, in the village vs outside the village). This procedure was implemented covering a two-week time window for non-durable household consumption, and a three-month time window for durable goods categories. To obtain total monthly retail expenditure, we multiply the bi-weekly expenditure on non-durables by a factor of 2 and divide durable good expenditure by a factor of 3 , and sum up across the 9 consumption categories. For expenditures on the new e-commerce option, we include both direct use of the terminal interface as well as remote usage by ordering deliveries to the terminal through the firm's app. The majority of terminal usage are done in person at the terminal rather than remotely. In most village cases, deliveries and pickups can be made at the terminal location ( 90 percent). In about 10 percent of cases, the logistics operators offered delivery to the home address too.

To construct total household income, our surveyors again used a pre-prepared spreadsheet to assist households in recording each of their individual income sources over the last month. We defined four income categories: farm earnings, non-farm earnings, remittances (money or in-kind) from family not living in the home, and all other income (e.g. pension, returns from savings, gifts). In addition, we recorded sector of activity and occupation categories for each economically active member of the household. To help household respondents recall and categorize earnings, surveyors used cards with detailed examples of income sources in each category and proceeded to collect each flow on the spreadsheet before filling out the final survey questionnaire in the presence of the household. Our measure of income per capita is the sum of all income sources in these four categories, divided by the number of household members. Our measure of income net of transfers subtracts gifts and remittances from family not living in the home. ${ }^{17}$ Our measure of income per capita net of costs subtracts the recorded household expenses used to generate the reported flows of income. The income variables exclude the market value of home production for own consumption. ${ }^{18}$ Including this as part of household income has no effect on the statistical zeros that we report in the analysis.

Finally, for households who were either replaced or added as part of our extended sample in the second round (from 28 to 38 households), we define $y_{h v}^{\text {Pre }}$ in specification (1) as the mean pre-treatment outcome of households living in the same zone (inner or outer) in the same village. The implicit assumption is that households were not induced to move within or across villages as a result of the program. As reported in appendix Table A.15, we find no evidence that households in treated villages are more or less likely to reside at the same address at endline. We also find no treatment effect on migration decisions of members within households.

\footnotetext{
${ }^{17}$ Remittances represent on average 13 percent of total household income in our sample.

${ }^{18}$ The market value of all food and beverages that the household produces for its own consumption amounts to on average less than 10 percent of household incomes.
} 


\section{F.6 Township-Level Data on Trade Market Access}

As part of our analysis of potential spillover effects on the control group in Appendix C, we estimate the fraction of a rural location's total trade market access that stems from trading relationships with other rural locations in the same county, as opposed to access to larger urban markets within and outside the county. To do this, we use geocoded township-level data from the Chinese Population Census in 2010, which contains information on the recorded population for each of roughly 45,000 township-level administrative units in China, ${ }^{19}$ the coordinates of the centroid of each of those units, the type of township-level unit (e.g. urban zones, rural townships) and data on the value added per rural and urban worker at the province level for 2010. See Appendix C for further discussion and details about the estimation.

\section{References}

Anderson, S. P., De Palma, A., \& Thisse, J. F. (1992). Discrete choice theory of product differentiation. MIT press.

Atkin, D., Faber, B., \& Gonzalez-Navarro, M. (2018). Retail globalization and household welfare: Evidence from mexico. Journal of Political Economy.

Disdier, A.-C., \& Head, K. (2008). The puzzling persistence of the distance effect on bilateral trade. The Review of Economics and Statistics, 90(1), 37-48.

Donaldson, D., \& Hornbeck, R. (2016). Railroads and American economic growth: A market access approach. The Quarterly Journal of Economics, 131(2), 799-858.

Feenstra, R. C. (1994). New product varieties and the measurement of international prices. American Economic Review, 84(1), 155-57.

Head, K., \& Mayer, T. (2014). Gravity equations: Workhorse, toolkit, and cookbook. In Handbook of International Economics (Vol. 4, pp. 131-195). Elsevier.

Kling, J. R., Liebman, J. B., \& Katz, L. F. (2007). Experimental analysis of neighborhood effects. Econometrica, 75(1), 83-119.

Miguel, E., \& Kremer, M. (2004). Worms: Identifying impacts on education and health in the presence of treatment externalities. Econometrica, 72(1), 159-217.

\footnotetext{
${ }^{19}$ This includes both the registered and non-registered population currently residing in the unit at the time of the census. Townships are the most disaggregated unit of observation that we can obtain the full census database for. In China's administrative hierarchy, townships are one layer above villages. In the countryside, townships include on average about 14 villages. In urban regions, township-level units are one level below urban districts.
} 